\title{
SPIRITS 16tn in NGC 3556: A Heavily Obscured and Low-luminosity Supernova at 8.8 Mpc
}

Jacob E. Jencson ${ }^{1,14}$ (iD, Mansi M. Kasliwal ${ }^{1}$ (D), Scott M. Adams ${ }^{1}$, Howard E. Bond ${ }^{2,3}$ (D), Ryan M. Lau ${ }^{1,4}$, Joel Johansson ${ }^{5}$ (iD, Assaf Horesh $^{6}$, Kunal P. Mooley ${ }^{7}$ (D), Robert Fender ${ }^{7}$, Kishalay De ${ }^{1}$, Dónal O’Sullivan ${ }^{1}$, Frank J. Masci ${ }^{8}$ (D), Ann Marie Cody ${ }^{9}$ (D), Nadia Blagorodnova ${ }^{1}$, Ori D. Fox ${ }^{3}$ (D), Robert D. Gehrz ${ }^{10}$ (iD), Peter A. Milne ${ }^{11}$ (D), Daniel A. Perley ${ }^{12,13}$ (iD), Nathan Smith ${ }^{11}$, and Schuyler D. Van Dyk $^{8}$ iD

${ }^{1}$ Cahill Center for Astronomy and Astrophysics, California Institute of Technology, Pasadena, CA 91125, USA; jj@astro.caltech.edu

${ }^{2}$ Department of Astronomy \& Astrophysics, Pennsylvania State University, University Park, PA 16802, USA

${ }^{3}$ Space Telescope Science Institute, 3700 San Martin Dr., Baltimore, MD 21218, USA

4 Jet Propulsion Laboratory, California Institute of Technology, 4800 Oak Grove Drive, Pasadena, CA 91109, USA

${ }^{5}$ Department of Physics and Astronomy, Division of Astronomy and Space Physics, Uppsala University, Box 516, SE-751 20 Uppsala, Sweden

${ }^{6}$ Racah Institute of Physics, The Hebrew University, Jerusalem 91904, Israel

${ }^{7}$ Department of Physics, Astrophysics, University of Oxford, Denys Wilkinson Building, Oxford OX1 3RH, UK

${ }^{8}$ Caltech/IPAC, Mailcode 100-22, Pasadena, CA 91125, USA

${ }^{9}$ NASA Ames Research Center, Moffet Field, CA 94035, USA

${ }^{10}$ Minnesota Institute for Astrophysics, School of Physics and Astronomy, 116 Church Street SE, University of Minnesota, Minneapolis, MN 55455, USA

${ }^{11}$ University of Arizona, Steward Observatory, 933 N. Cherry Avenue, Tucson, AZ 85721, USA

${ }^{12}$ Dark Cosmology Centre, Niels Bohr Institute, University of Copenhagen, Juliane Maries Vej 30, DK-2100 Copenhagen $\emptyset$, Denmark

${ }^{13}$ Astrophysics Research Institute, Liverpool John Moores University, IC2, Liverpool Science Park, 146 Brownlow Hill, Liverpool L3 5RF, UK Received 2018 February 24; revised 2018 June 13; accepted 2018 June 25; published 2018 August 7

\begin{abstract}
We present the discovery by the SPitzer InfraRed Intensive Transients Survey (SPIRITS) of a likely supernova (SN) in NGC 3556 (M108) at only $8.8 \mathrm{Mpc}$ that was not detected by optical searches. A luminous infrared (IR) transient at $M_{[4.5]}=-16.7 \mathrm{mag}$ (Vega), SPIRITS 16tn is coincident with a dust lane in the inclined, star-forming disk of the host. Using observations in the IR, optical, and radio, we attempt to determine the nature of this event. We estimate $A_{V} \approx 8-9$ mag of extinction, placing it among the three most highly obscured IR-discovered SNe. The [4.5] light curve declined at a rate of $0.013 \mathrm{mag} \mathrm{day}^{-1}$, and the [3.6]-[4.5] color increased from 0.7 to $\gtrsim 1.0$ mag by 184.7 days post discovery. Optical/IR spectroscopy shows a red continuum but no clearly discernible features, preventing a definitive spectroscopic classification. Radio observations constrain the radio luminosity of SPIRITS 16tn to $L_{\nu} \lesssim 10^{24} \mathrm{erg} \mathrm{s}^{-1} \mathrm{~Hz}^{-1}$ between 3 and $15 \mathrm{GHz}$, excluding many varieties of core-collapse SNe. An SN Ia is ruled out by the observed IR color and lack of spectroscopic features from Fe-peak elements. SPIRITS 16tn was fainter at [4.5] than typical stripped-envelope SNe by $\approx 1$ mag. Comparison of the spectral energy distribution to SNe II suggests that SPIRITS 16tn was both highly obscured and intrinsically dim, possibly akin to the low-luminosity SN $2005 \mathrm{cs}$. We infer the presence of an IR dust echo powered by an initial peak luminosity of the transient of $5 \times 10^{40} \mathrm{erg} \mathrm{s}^{-1} \lesssim L_{\text {peak }} \lesssim 4 \times 10^{43} \mathrm{erg} \mathrm{s}^{-1}$, consistent with the observed range for SNe II. This discovery illustrates the power of IR surveys to overcome the compounding effects of visible extinction and optically subluminous events in completing the inventory of nearby SNe.
\end{abstract}

Key words: dust, extinction - galaxies: individual (NGC 3556) - supernovae: general - supernovae: individual (SPIRITS 16tn) - surveys

\section{Introduction}

The discovery and characterization of core-collapse supernovae (CCSNe), bursts of light heralding the explosive deaths of stars with initial mass $\gtrsim 8 M_{\odot}$, have been largely driven in recent years by several large optical time-domain surveys, many specifically dedicated to the identification of transients. While such searches have been hugely successful, now discovering hundreds of SNe every year, a primary limitation is the susceptibility of visible photons to extinction by intervening dust. CCSNe in particular, often associated with the dense and dusty star-forming regions of late-type galaxies, may be subject to significant host extinction.

The measurement of the CCSN rate from optical surveys is an important probe of star formation and the fate of massive stars. However, these measurements only yield lower limits, as some $\mathrm{SNe}$ are missed owing to obscuration (e.g., Grossan

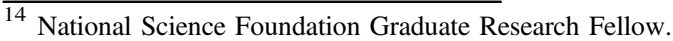

et al. 1999; Maiolino et al. 2002; Cresci et al. 2007). In particular, Horiuchi et al. (2011) claim that half of all supernovae are missing across redshifts from $0<z<1$, termed the "supernova rate problem" and possibly indicating a large population of hidden or intrinsically dim SNe. Cappellaro et al. (2015) have challenged this claim, however, finding full agreement between CCSN rates and revised measurements of the cosmic star formation history. Still, Mannucci et al. (2007) estimate that $5 \%-10 \%$ of CCSNe locally are inaccessible to optical searches, rising steeply to $>30 \%$ beyond $z=1$. More recently, Mattila et al. (2012) find empirically that $\sim 20 \%$ of SNe locally, growing to $\sim 40 \%$ by $z=1$, may be missed by optical searches owing only to obscuration by dust. The deep, galaxy-targeted $D<40 \mathrm{Mpc}$ (DTL40) SN search recently reported, for example, the discovery of the obscured Type II SN DLT16am (SN 2016ija) in the nearby, edge-on galaxy NGC 1532 with $A_{V} \approx 6 \mathrm{mag}$ (Tartaglia et al. 2018). Further confounding the debate, recent 
studies suggest that CCSNe may even be overproduced in the local $11 \mathrm{Mpc}$ volume (Botticella et al. 2012; Horiuchi et al. 2013; Xiao \& Eldridge 2015) compared to $\mathrm{H} \alpha$ - and ultraviolet(UV-) inferred star formation rates. Any CCSNe missed in the nearest galaxies only increase this tension.

Transient surveys at infrared (IR) wavelengths can overcome the limitations of optical searches introduced by the effects of extinction. A number of searches in the near-IR have focused specifically on the dense, highly star-forming, heavily extinguished environments of luminous and ultraluminous infrared galaxies (LIRGs and ULIRGs), where the $\mathrm{SN}$ rates are expected to be high, of order one per year (Mattila \& Meikle 2001). Such surveys, using seeing-limited imaging (e.g., Mannucci et al. 2003; Miluzio et al. 2013) or high-resolution imaging from space or with ground-based adaptive optics to probe the densest nuclear regions of these galaxies (e.g., Cresci et al. 2007; Mattila et al. 2007; Kankare et al. 2008, 2012; Kool et al. 2018), have now uncovered 16 CCSNe in (U)LIRGs.

The InfraRed Array Camera (IRAC; Fazio et al. 2004) aboard the Spitzer Space Telescope (Werner et al. 2004; Gehrz et al. 2007), in the 3.6 and $4.5 \mu \mathrm{m}$ imaging bands (hereafter [3.6] and [4.5]), where the effects of extinction are minimal, is sensitive to even the most highly obscured events, up to $A_{V} \approx 100 \mathrm{mag}$ at $20 \mathrm{Mpc}$. Since 2014 December, the SPitzer InfraRed Intensive Transients Survey (SPIRITS; PIDs 11063, 13053; PI M. Kasliwal, Kasliwal et al. 2017) has been conducting an ongoing monitoring campaign of nearby galaxies $(D \lesssim 20 \mathrm{Mpc})$ for transients with Spitzer/IRAC at [3.6] and [4.5]. An example of the importance of IR surveys was demonstrated in Jencson et al. (2017), where we reported the discovery of two obscured SNe in IC 2163, SPIRITS 14buu and SPIRITS 15C, missed by optical searches despite their proximity to Earth and only moderate amounts of extinction $\left(A_{V} \approx 1.5-2.2 \mathrm{mag}\right)$.

Beyond the ability to discover CCSNe hidden by dust, midIR observations offer important diagnostics of the explosions and their circumburst environments. Mid-IR emission may be produced in $\mathrm{SNe}$ as thermal emission from the photosphere of the explosions, and it also traces the presence of warm dust in the system. This dust may be newly formed in the ejecta or in the rapidly cooling, post-shock material of the explosion. Alternatively, preexisting circumburst dust, possibly formed in the pre-SN stellar wind or an eruptive mass-loss event of the progenitor star, may be heated by the luminous SN peak, producing an "IR echo" owing to light-travel time effects (e.g., Bode \& Evans 1980; Dwek 1983; Mattila et al. 2008). The multifaceted effects of dust, either newly formed or preexisting, on the mid-IR emission of SNe have been studied in numerous works (see, e.g., Kotak et al. 2009; Fox et al. 2010, 2011; Szalai \& Vinkó 2013). Most recently, Tinyanont et al. (2016) and Szalai et al. (2018) presented systematic studies of CCSNe observed in the mid-IR with Spitzer, finding remarkable diversity in the growing sample of well-characterized events at these wavelengths.

Here we report the discovery of SPIRITS 16tn, a likely highly obscured CCSN at only $8.8 \mathrm{Mpc}$ in the nearby spiral galaxy NGC 3556 (M108). In Section 2, we describe the discovery and follow-up observations of this event using both space- and ground-based facilities in the optical, IR, and radio. In Section 3, we describe our analysis of the data, including constraints on the progenitor luminosity from archival, pre-explosion Hubble Space Telescope (HST) imaging (Section 3.1), analysis of the light curves and color evolution (Section 3.2), evolution of the spectral energy distribution (SED), and constraints on the extinction and dust emission (Section 3.3). In Section 4, we discuss the overall properties of SPIRITS 16th and our interpretation of the observations in the context of well-studied $\mathrm{SNe}$ and other types of luminous IR transients. We present our conclusions in Section 5.

\section{SPIRITS Discovery and Follow-up Observations}

\subsection{Spitzer/IRAC Discovery in NGC 3556}

During the ongoing monitoring campaign of nearby galaxies with SPIRITS, we observed the star-forming galaxy NGC 3556 with Spitzer/IRAC at [3.6] and [4.5] at 10 epochs between UT 2014 January 18.4 and 2016 August 15.0. Image subtraction was performed using archival images from 2011 February 7.6 as references (observed as part of the Spitzer Survey of Stellar Structure in Galaxies, S ${ }^{4}$; PID 61065; PI K. Sheth; Sheth et al. 2010). For details on our image subtraction pipeline see Kasliwal et al. (2017). A new transient source, designated SPIRITS 16tn, was detected in both the [3.6] and [4.5] images on 2016 August 15.0 (MJD = 57,615.0; Jencson et al. 2016). Throughout this paper, we refer to the phase as the number of days since the earliest detection of SPIRITS 16tn on this date. We detect no significant variability at the location of SPIRITS 16tn in any of the prior Spitzer/IRAC images compared to the reference frame. We show the [4.5] discovery images in the middle row of Figure 1, along with mosaicked gri imaging of the field from the Sloan Digital Sky Survey Date Release 12 (SDSS-DR12; Eisenstein et al. 2011; Alam et al. 2015) in the top panel, showing the location of SPIRITS 16tn in a dust lane in the disk of NGC 3556. This galaxy was also the host of the probable Type II SN 1969B (Ciatti \& Barbon 1971).

\subsubsection{Host Distance and Galactic Extinction}

SPIRITS 16tn was discovered at a right ascension and declination of $11^{\mathrm{h}} 11^{\mathrm{m}} 20^{\mathrm{s}} 40,+55^{\circ} 40^{\prime} 177^{\prime \prime} 3$ (J2000). Located $89 . " 9$ from the center of the star-forming galaxy NGC 3556, the position of SPIRITS 16tn is coincident with a dust lane in the disk

$\mathrm{NED}^{15}$ lists 17 individual distance estimates to NGC 3556 , with a median value of $\mu=29.71 \mathrm{mag}$ and standard deviation of $0.67 \mathrm{mag}$. Throughout this work, we adopt the most recent value from Sorce et al. (2014) of $\mu=29.72 \pm 0.41 \mathrm{mag}$ $(D \approx 8.8 \mathrm{Mpc})$. This estimate is based on the mid-IR TullyFisher relation using the [3.6] micron flux with color and selection bias corrections. The redshift of NGC 3556 is $z=0.002332\left(v=699 \mathrm{~km} \mathrm{~s}^{-1}\right.$; Shostak 1975).

We assume Galactic extinction along the line of sight to NGC 3556 of $A_{V}=0.046$ mag from the Schlafly \& Finkbeiner (2011) recalibration of the Schlegel et al. (1998) IR-based dust map assuming a Fitzpatrick (1999) extinction law with $R_{V}=3.1$. Furthermore, for all other considerations of the possible extinction to SPIRITS 16tn throughout this work, including any foreground host extinction, we assume the Fitzpatrick (1999) Milky Way extinction curve with $R_{V}=3.1$ unless otherwise noted.

\footnotetext{
${ }^{15}$ The NASA/IPAC Extragalactic Database (NED) is operated by the Jet Propulsion Laboratory, California Institute of Technology, under contract with the National Aeronautics and Space Administration.
} 


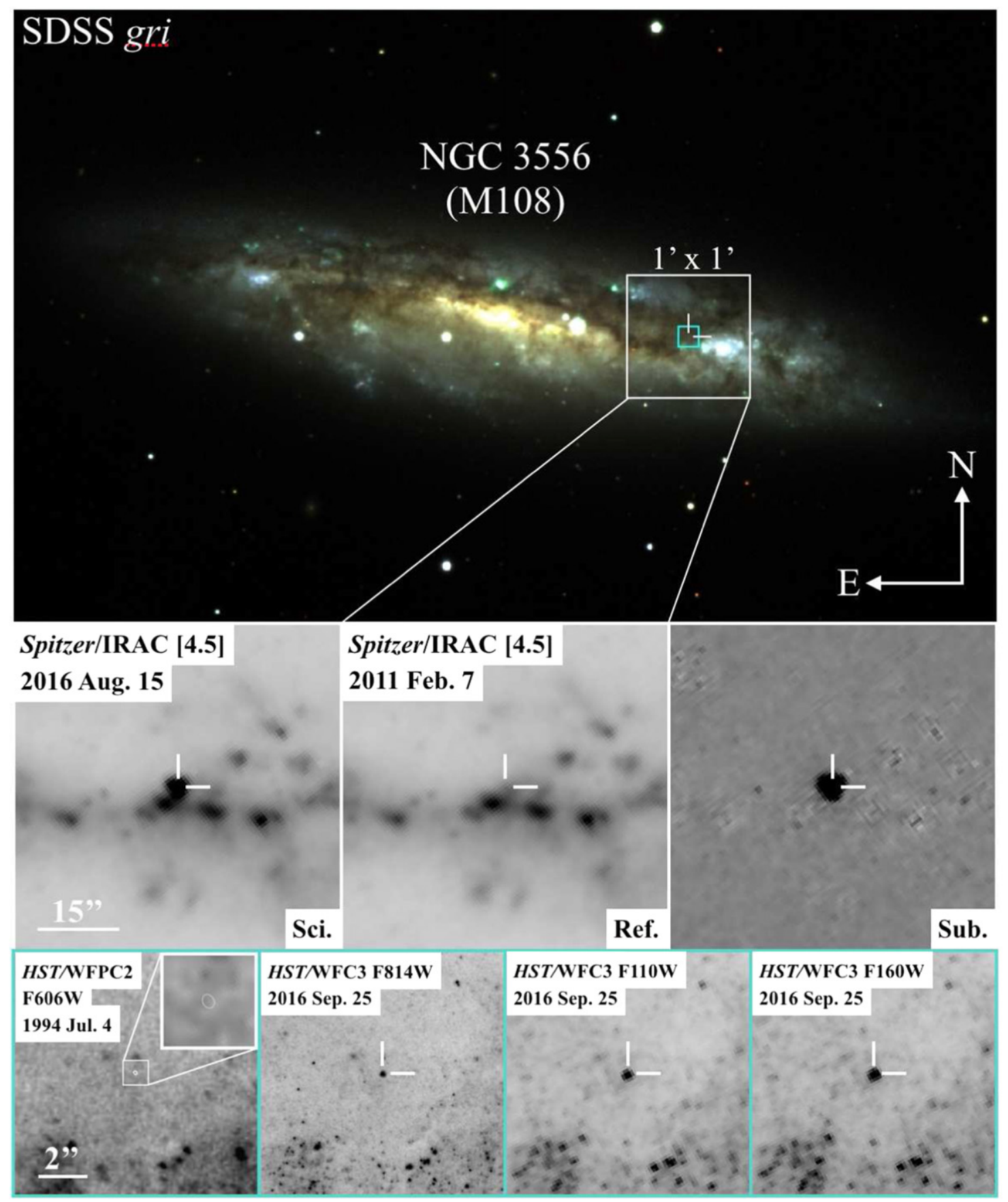

Figure 1. Top panel: color-composite SDSS imaging of NGC 3556 (M108) in three filters ( $g$ in blue, $r$ in green, and $i$ in red). The location of SPIRITS 16tn in a dust lane of NGC 3556 is indicated by the white crosshairs. The middle row shows the $1^{\prime} \times 1^{\prime}$ region indicated by the white zoom-in box in the top panel. From left to right, we show the Spitzer/IRAC [4.5] discovery science frame of SPIRITS 16tn from 2016 August 15, the archival reference image from 2011 February 7, and the science-reference subtraction image, clearly showing the new transient source. In the bottom row, we show the $10^{\prime \prime} \times 10^{\prime \prime}$ region indicated by the cyan box in the top panel. In the leftmost panel, we show the archival HST/WFPC2 F606W image. The white ellipse (shown more clearly in the $1^{\prime \prime} \times 1^{\prime \prime}$ zoom-in in the upper right corner of this panel) indicates the $10 \sigma$ uncertainty on the position of SPIRITS 16tn. In the three rightmost panels of the bottom row, we show the post-discovery HST/WFC 3 F814W, F110W, and F160W imaging of SPIRITS 16tn.

\subsection{Follow-up Imaging}

In this section, we describe our space- and ground-based imaging follow-up efforts to characterize SPIRITS 16tn.

\subsubsection{Space-based}

Since its discovery, we continued to monitor SPIRITS 16tn with Spitzer/IRAC at [3.6] and [4.5] as part of the SPIRITS program. Image subtraction was performed on all subsequent epochs, as described in Section 2.1. Photometry was performed on the reference-subtracted images using a 4 mosaicked pixel (2". 4) aperture and a background annulus from 4 to 12 pixels (2!"4-7!'2). The extracted flux was multiplied by aperture corrections of 1.215 and 1.233 for [3.6] and [4.5], respectively, as described in the IRAC instrument handbook. ${ }^{16}$ Fluxes then

\footnotetext{
$\overline{16}$ http://irsa.ipac.caltech.edu/data/SPITZER/docs/irac/ iracinstrumenthandbook/
} 
were converted to Vega system magnitudes using the handbook-defined zero-magnitude fluxes for each IRAC channel. At discovery, our photometry gives $[4.5]=13.04 \pm 0.05$ mag $\left(M_{[4.5]}=-16.7 \mathrm{mag} ; \lambda L_{\lambda}=1.3 \times 10^{7} L_{\odot}\right)$.

We triggered observations with the Ultra-violet/Optical Telescope (UVOT; Roming et al. 2005) on board the Neil Gehrels Swift Observatory (Gehrels et al. 2004; Nousek 2004) on 2016 August 29.1. No source was detected in the $U-, B$-, and $V$-band images with integration times of 540, 580, and $540 \mathrm{~s}$, respectively (Adams et al. 2016a). We derived $5 \sigma$ limiting magnitudes of $V>19.9 \mathrm{mag}, B>20.2 \mathrm{mag}$, and $U>19.9$ mag. The extreme $V-[4.5] \gtrsim 6.9 \mathrm{mag}$ color indicates that SPIRITS 16tn is likely highly obscured.

We observed SPIRITS 16tn on 2016 September 25, $t=42$ days, with the Wide Field Camera 3 (WFC3) on the $H S T$ in the UVIS channel with the F814W filter and the IR channel with the F110W and F160W filters. These observations were part of our Cycle 23 Target of Opportunity program to observe SPIRITS transients (GO-14258; PI: H. Bond). All three images are shown in the bottom row of Figure 1. The photometry and limits from our space-based follow-up effort are listed in Table 1 and shown in Figure 2.

\subsubsection{Ground-based}

At the time of its discovery, SPIRITS 16tn was inaccessible for ground-based observing except at high latitudes. We began ground-based follow-up of SPIRITS 16tn from Palomar Observatory in 2016 October, approximately 2 months after discovery. We obtained near-IR images of SPIRITS 16tn in $J H K_{s}$ with the Wide Field Infrared Camera (WIRC; Wilson et al. 2003) on the Palomar 200-inch Hale telescope (P200) at several epochs, employing large dithers approximately every minute to allow for accurate subtraction of the bright near-IR sky background. Flat-fielding, background subtraction, astrometric alignment, and final stacking of images in each filter were performed using a custom pipeline.

Additional near-IR $H$ - and $K_{s}$-band imaging was obtained with the Wide Field Camera (WFCAM; Casali et al. 2007) on the United Kingdom Infrared Telescope (UKIRT) at Mauna Kea Observatories. We obtained simultaneous optical/near-IR rizYJH with the Reionization and Transients InfraRed camera (RATIR; Butler et al. 2012) on the $1.5 \mathrm{~m}$ Johnson Telescope at the Mexican Observatorio Astronomico Nacional on the Sierra San Pedro Martir in Baja California, Mexico (Watson et al. 2012).

We obtained one epoch of optical ( $i$-band) imaging with the Spectral Energy Distribution Machine (SEDM; Blagorodnova et al. 2018) on the fully automated Palomar 60-inch telescope (P60; Cenko et al. 2006) and an epoch of $g$ - and I-band imaging with the Low-Resolution Imaging Spectrometer (LRIS; Oke et al. 1995) on the Keck I Telescope on Maunakea.

Photometry was performed by simultaneously fitting the point-spread function of the transient, measured using field stars, and background, modeled using low-order polynomials. The photometric zero-point in each image was obtained by performing photometry on stars of known magnitude in the field. For the near-IR $J H K$ images we selected 10 bright, isolated Two Micron All Sky Survey (2MASS) stars, and for $Y$ band we adopt the conversion from 2MASS used for WFCAM/UKIRT from Hodgkin et al. (2009). For optical images, we used 12 SDSS stars, adopting the conversions of
Jordi et al. (2006) to convert from the Sloan ugriz system to $U B V R I$ magnitudes where necessary.

We examined the location of SPIRITS 16tn in a deep $z$-band image of NGC 3556 from 2017 May 25.0, taken with the CCD Mosaic imager on the $4 \mathrm{~m}$ Mayall Telescope at Kitt Peak National Observatory (KPNO) as part of the Mayall $z$-band Legacy Survey (MzLS). We derive a limit on the flux from the transient of $z>22.5 \mathrm{mag}$, providing our most stringent constraint on the explosion date of SPIRITS 16th at 82.0 days before the first detection.

We list all of our photometry of SPIRITS 16tn in Table 1. For nondetections we list $5 \sigma$ upper limits, where we estimated $\sigma$ as the standard deviation of the pixel values near the transient position to account for uncertain variations in the background flux from the host galaxy. Our light curves of SPIRITS 16tn are shown in Figure 2. Additionally, Shappee et al. (2016) reported pre-discovery limits from nondetections of SPIRITS 16tn in the All-Sky Automated Survey for Supernovae (ASAS-SN) between UT 2016 May 16.3 and 2016 June 10.3, constraining optical emission from SPIRITS 16tn to $V>17.81$ mag at $t=-65.7$ days. Vinko et al. (2016) also reported observations in the Sloan $i$ filter of SPIRITS 16tn on 2016 September 1.8, placing a limit on the flux from the transient of $i>18.7$ mag at $t=17.8$ days. We show these limits along with our own photometry in Figure 2. As reported, these limits do not further constrain the extinction or explosion date beyond the data newly presented here.

\subsection{Spectroscopy}

We obtained optical spectroscopy of SPIRITS 16tn with Keck/LRIS on 2017 November 2 ( $t=79$ days post discovery). We used the D560 dichroic to split the light between the red and blue sides, and we used the 400/8500 grating on the red side and the $300 / 3400$ grism on the blue side. We obtained one $1800 \mathrm{~s}$ integration on the blue side and two $860 \mathrm{~s}$ integrations on the red side. Spectroscopic reductions were performed using the analysis pipeline LPIPE. ${ }^{17}$ A weak trace is visible at the position of the transient on the red-side camera. The low signalto-noise ratio $(\mathrm{S} / \mathrm{N})$ 1D extracted spectrum was flux-calibrated using observations of the standard star Feige 34 from the same night. The Keck/LRIS optical spectrum is shown in Figure 3.

We observed SPIRITS 16tn with the Gemini Near-Infrared Spectrograph (GNIRS) on the $8.1 \mathrm{~m}$ Gemini North Telescope on the summit of Maunakea in Hawaii through Gemini Fast Turnaround program GN-2016B-FT-25. We obtained two epochs $^{18}$ of near-IR cross-dispersed (XD; multi-order) spectroscopy on 2016 December 29 ( $t=136$ days) and 2017 January $9(t=147$ days $)$ using a 0.45 arcsec wide slit with the 32 line $\mathrm{mm}^{-1}$ grating and the short blue camera with its crossdispersing prism for a spectra resolution of $R=1200$. In this configuration, a spectrum of the entire near-IR region $(0.85-2.5 \mu \mathrm{m})$ is obtained at once. The observations were carried out using $300 \mathrm{~s}$ exposures, with the target nodded along the slit between frames to allow for accurate subtraction of the sky background. We obtained a total of 70 minutes of integration during the first epoch and 50 minutes during the

\footnotetext{
17 Software available at http://www.astro.caltech.edu/ dperley/programs / lpipe.html.

${ }^{18}$ Our observations were submitted as a single observation to the Gemini queue, but the execution of our program was split between two separate dates instead, possibly due to deteriorated weather conditions.
} 
Table 1

Photometry of SPIRITS 16tn

\begin{tabular}{|c|c|c|c|c|c|c|}
\hline UT Date & MJD & $\begin{array}{l}\text { Phase }^{\mathrm{a}} \\
\text { (days) }\end{array}$ & Tel./Inst. & Band & $\begin{array}{l}\text { Apparent Magnitude } \mathrm{e}^{\mathrm{b}, \mathrm{c}} \\
\text { (mag) }\end{array}$ & $\begin{array}{l}\text { Absolute Magnitude }{ }^{\mathrm{c}, \mathrm{d}} \\
(\mathrm{mag})\end{array}$ \\
\hline 1999 Jul 04 & 51,363 & -6252 & $H S T /$ WFPC2 & F606W & $>24.5$ & $>-5.2$ \\
\hline 2016 Mar 03.6 & $57,450.6$ & -164.4 & Spitzer/IRAC & {$[3.6]$} & $>17.8$ & $>-11.9$ \\
\hline 2016 May 25.0 & $57,533.0$ & -82.0 & KPNO-4 m & $z$ & $>22.5$ & $>-7.2$ \\
\hline 2016 Aug 15.0 & $57,615.0$ & 0.0 & Spitzer/IRAC & {$[3.6]$} & $13.71(0.05)$ & -16.0 \\
\hline 2016 Aug 15.0 & $57,615.0$ & 0.0 & Spitzer/IRAC & {$[4.5]$} & $13.04(0.05)$ & -16.7 \\
\hline 2016 Aug 29.0 & $57,629.0$ & 14.0 & Swift/UVOT & V & $>20.5$ & $>-9.2$ \\
\hline 2016 Sep 25.9 & $57,656.9$ & 41.9 & $H S T / \mathrm{WFC} 3$ & F814W & $21.68(0.03)$ & -8.0 \\
\hline 2016 Sep 25.9 & $57,656.9$ & 41.9 & $H S T / \mathrm{WFC} 3$ & F110W & $19.76(0.02)$ & -10.0 \\
\hline 2016 Sep 25.9 & $57,656.9$ & 41.9 & $H S T / \mathrm{WFC} 3$ & F160W & $18.64(0.02)$ & -11.1 \\
\hline 2016 Oct 11.5 & $57,672.5$ & 57.5 & P200/WIRC & $K_{s}$ & $16.9(0.1)$ & -12.8 \\
\hline 2016 Nov 09.4 & $57,701.4$ & 86.4 & $1.5 \mathrm{~m} / \mathrm{RATIR}$ & $i$ & $>19.6$ & $>-10.1$ \\
\hline 2016 Nov 09.4 & $57,701.4$ & 86.4 & $1.5 \mathrm{~m} / \mathrm{RATIR}$ & $z$ & $>19.7$ & $>-10.0$ \\
\hline 2016 Nov 09.4 & $57,701.4$ & 86.4 & $1.5 \mathrm{~m} / \mathrm{RATIR}$ & $Y$ & $>18.6$ & $>-11.1$ \\
\hline 2016 Nov 09.4 & $57,701.4$ & 86.4 & $1.5 \mathrm{~m} / \mathrm{RATIR}$ & $J$ & $>18.0$ & $>-11.7$ \\
\hline 2016 Nov 09.4 & $57,701.4$ & 86.4 & $1.5 \mathrm{~m} / \mathrm{RATIR}$ & $H$ & $>17.5$ & $>-12.2$ \\
\hline 2016 Nov 10.4 & $57,702.4$ & 87.4 & $1.5 \mathrm{~m} / \mathrm{RATIR}$ & $r$ & $>19.9$ & $>-9.8$ \\
\hline 2016 Nov 10.4 & $57,702.4$ & 87.4 & $1.5 \mathrm{~m} / \mathrm{RATIR}$ & $i$ & $>19.8$ & $>-9.9$ \\
\hline 2016 Nov 10.4 & $57,702.4$ & 87.4 & $1.5 \mathrm{~m} / \mathrm{RATIR}$ & $z$ & $>19.7$ & $>-10.0$ \\
\hline 2016 Nov 10.4 & $57,702.4$ & 87.4 & $1.5 \mathrm{~m} / \mathrm{RATIR}$ & $Y$ & $>18.8$ & $>-10.9$ \\
\hline 2016 Nov 10.4 & $57,702.4$ & 87.4 & $1.5 \mathrm{~m} / \mathrm{RATIR}$ & $J$ & $>18.2$ & $>-11.5$ \\
\hline 2016 Nov 10.4 & $57,702.4$ & 87.4 & $1.5 \mathrm{~m} / \mathrm{RATIR}$ & $H$ & $>17.6$ & $>-12.1$ \\
\hline 2016 Dec 14.7 & $57,736.7$ & 121.7 & UKIRT & $H$ & $18.9(0.3)$ & -10.8 \\
\hline 2017 Apr 09.8 & $57,852.8$ & 237.8 & Spitzer/IRAC & [3.6] & $>17.7$ & $>-12.0$ \\
\hline 2017 Apr 09.8 & $57,852.8$ & 237.8 & Spitzer/IRAC & [4.5] & $17.2(0.2)$ & -12.5 \\
\hline 2017 May 03.3 & $57,876.3$ & 261.3 & P200/WIRC & $J$ & $>20.0$ & $>-9.7$ \\
\hline 2017 May 03.3 & $57,876.3$ & 261.3 & P200/WIRC & $H$ & $>19.2$ & $>-10.5$ \\
\hline 2017 May 03.3 & $57,876.3$ & 261.3 & P200/WIRC & $K_{s}$ & $>18.5$ & $>-11.2$ \\
\hline 2017 Jul 10.3 & $57,944.3$ & 329.3 & Spitzer/IRAC & {$[3.6]$} & $>17.6$ & $>-12.1$ \\
\hline 2017 Jul 10.3 & $57,944.3$ & 329.3 & Spitzer/IRAC & {$[4.5]$} & $>17.5$ & $>-12.2$ \\
\hline 2018 Feb 21.3 & $58,170.3$ & 555.3 & Spitzer/IRAC & [3.6] & $>17.9$ & $>-11.8$ \\
\hline 2018 Feb 21.3 & $58,170.3$ & 555.3 & Spitzer/IRAC & {$[4.5]$} & $>17.9$ & $>-11.8$ \\
\hline
\end{tabular}

Notes.

${ }^{a}$ Phase is number of days since the earliest detection of this event on 2016 August 15.0 (MJD = 57,615.0).

${ }^{\mathrm{b}}$ Vega magnitudes, except for the griz bands, which are AB magnitudes on the SDSS system. $1 \sigma$ uncertainties are given in parentheses.

c $5 \sigma$ limiting magnitudes are given for nondetections.

d Absolute magnitudes corrected for Galactic extinction for NGC 3556 from NED.

second. Baseline calibrations were also obtained, including observations of A0V stars at similar airmass immediately before/after the science observations as near-IR standards for flux calibration and telluric corrections.

Reductions, including detector pattern noise cleaning, radiation event removal, flat-fielding, background subtraction, spatial distortion corrections, wavelength calibration, and 1D extractions, were perform using standard tasks in the Gemini IRAF ${ }^{19}$ package following the procedures outlined on the Gemini webpage. ${ }^{20}$ In

\footnotetext{
19 IRAF is distributed by the National Optical Astronomy Observatory, which is operated by the Association of Universities for Research in Astronomy (AURA) under a cooperative agreement with the National Science Foundation. ${ }^{20}$ Procedures for reducing GNIRS XD spectra are found at http://www.gemini. edu/sciops/instruments/gnirs/data-format-and-reduction/reducing-xd-spectra
} 


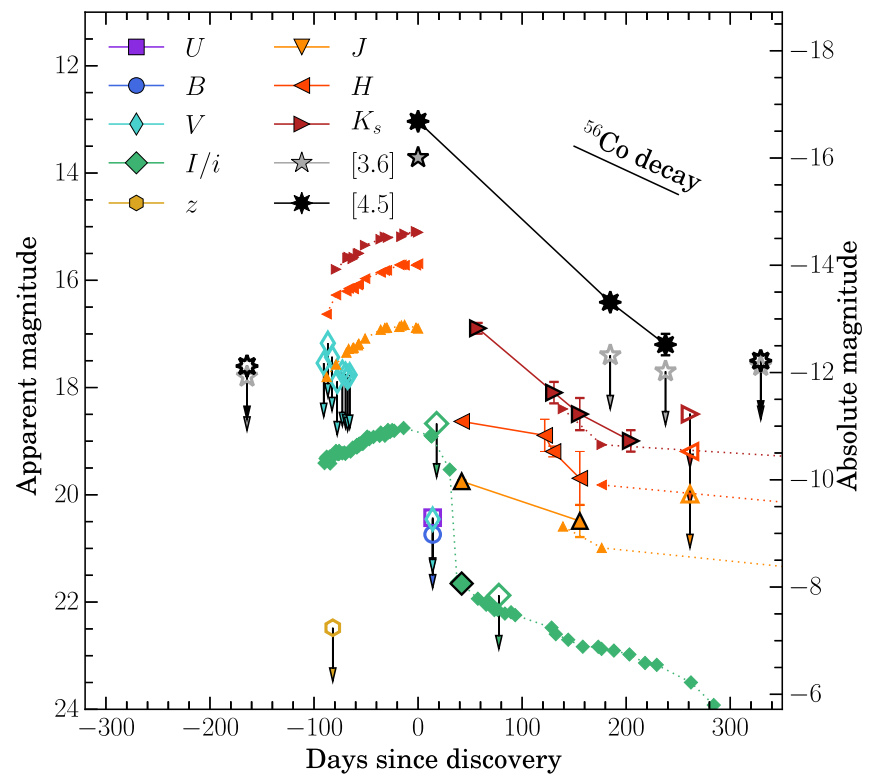

Figure 2. Multiband light curves of SPIRITS 16tn, corrected for Galactic extinction only, are shown as the large filled symbols, with $5 \sigma$ upper limits from nondetections indicated by open symbols with downward-pointing arrows. We also show the ASAS-SN V-band limits (Shappee et al. 2016) and Sloan $i$-band limit (Vinko et al. 2016) as reported. Small symbols are the corresponding light curves of the low-luminosity SN 2005cs from Pastorello et al. (2006, 2009), shifted to the distance of NGC 3556 plus an additional $\Delta m=0.7 \mathrm{mag}$, and reddened by $E(B-V)=2.5 \mathrm{mag}$. The black solid line indicates the expected decline rate for a light curve powered by the radioactive decay of ${ }^{56}$ Co (see, e.g., Gehrz 1988; Gehrz \& Ney 1990).

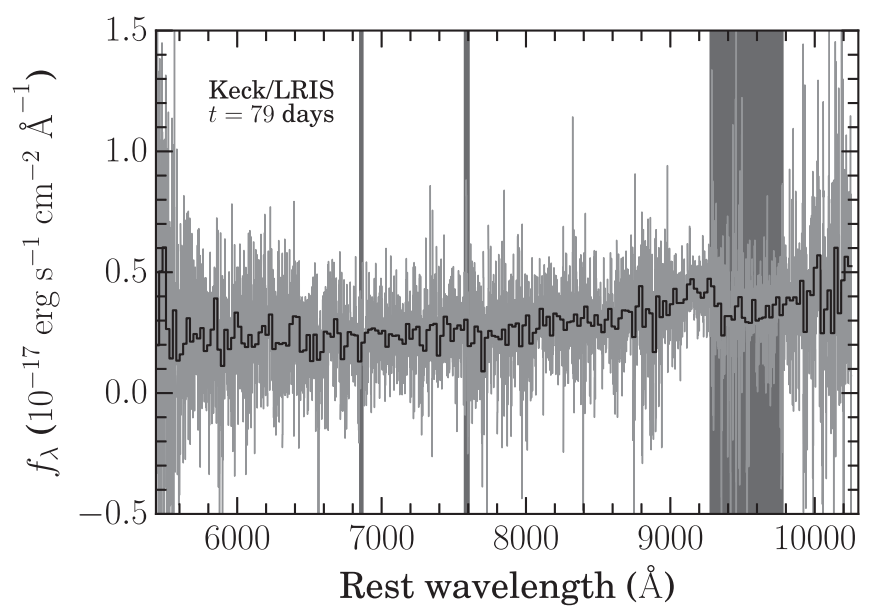

Figure 3. Optical spectrum of SPIRITS 16tn from Keck/LRIS taken on 2016 November $2(t=79$ days $)$ in the rest frame of the host galaxy, NGC 3556 $(z=0.002332)$. The data are shown in light gray, and the median-binned spectrum with a bin size of 20 pixels is overplotted in black. Regions affected by telluric absorption features are indicated by the dark-gray vertical bands.

the reduced $2 \mathrm{D}$ spectra, a faint trace was visible at the position of SPIRITS 16tn in spectral orders 3 and 4, corresponding to the $K$ and $H$ spectral regions, respectively.

Corrections for the strong near-IR telluric absorption features and flux calibrations were performed using the IDL tool xtellcor developed by Vacca et al. (2003). For the first epoch, a detector bias fault occurred during the science target observations, after which the target had to be reacquired. We reduced the two groups of data separately, using the star HIP 53735, observed immediately preceding SPIRITS 16tn, as the A0V flux standard for the first group and HIP 56147, observed immediately after, for the second. The two telluric-corrected, flux-calibrated spectra were then averaged. For the second epoch we again used HIP 53735. Our Gemini/GNIRS spectra of SPIRITS 16tn are shown in Figure 4.

We note that we did not attempt to subtract the contribution from the host-galaxy background from our optical/near-IR spectra, which may be significant for our late-time observations as the transient fades.

\subsubsection{Host Spectroscopy}

The Palomar Cosmic Web Imager (PCWI; Matuszewski et al. 2010) is an integral field spectrograph mounted on the Cassegrain focus of the 200-inch Hale telescope at Palomar Observatory. The instrument has a field of view of $40^{\prime \prime} \times 60^{\prime \prime}$ divided across 24 slices with dimensions of $40^{\prime \prime} \times 2$." 5 each. The spectrograph uses an $R \sim 5000$ volume phase holographic grating (in the red filter) to achieve an instantaneous bandwidth of $\approx 550 \AA$. A complete description of the instrument, observing approach, and data analysis methodology can be found in Martin et al. (2014).

We observed the host region of SPIRITS 16tn with PCWI (centered at the location of the transient) on 2017 October 18 in order to characterize the star formation rate in the transient environment. The instrument was configured to a central wavelength of $6630 \AA$, covering the wavelength range from approximately 6400 to $6900 \mathrm{~A}$. We obtained one $600 \mathrm{~s}$ exposure of the transient region with the instrument oriented to a position angle of $270^{\circ}$ (slices oriented in the north-south direction) followed by one $600 \mathrm{~s}$ background sky exposure of a nearby field with no bright sources.

We also obtained calibration images including arc lamp spectra, dome flats, and a standard-star spectrum. The 2D spectra were sliced, rectified, spatially aligned, and wavelength-calibrated using the calibration images to produce data cubes for each sky exposure, sampled at (R.A., decl., $\lambda$ ) intervals of $(2$ !' 6,0 ! $6,0.22 \AA)$. The sky background cube was subtracted from the source cube to remove the sky emission lines, followed by flux calibration using the standard star Feige 15. This produces the final flux-calibrated spectral cube of the $40^{\prime \prime} \times 60^{\prime \prime}$ region centered at the location of the transient.

\subsection{Radio Observations}

We observed SPIRITS 16tn in the radio with the Karl G. Jansky Very Large Array (VLA) at two epochs on 2016 September $9.0(t=19.0$ days $)$ in the $S, C$, and $X$ bands $(3,6$, and $10 \mathrm{GHz}$, respectively) and 2017 January $12.4(t=149.4$ days $)$ in the $C$ and $K u$ bands (10 and $15.5 \mathrm{GHz}$, respectively). The data were reduced using standard imaging techniques for the VLA in CASA. We also obtained radio imaging at $15 \mathrm{GHz}$ with the Arcminute Microkelvin Imager Large Array (AMI-LA) on 2017 September 2-5 ( $t=17-20$ days). The AMI-LA data were processed (RFI excision and calibration) with a fully automated pipeline, AMI-REDUCE (e.g., Davies et al. 2009; Perrott et al. 2013), and later imported and imaged in CASA. SPIRITS 16tn was undetected at all epochs and frequencies in the radio, and we provide a summary of our limits on the observed fluxes and radio luminosities of the source in Table 2. The $5 \sigma$ limits are 


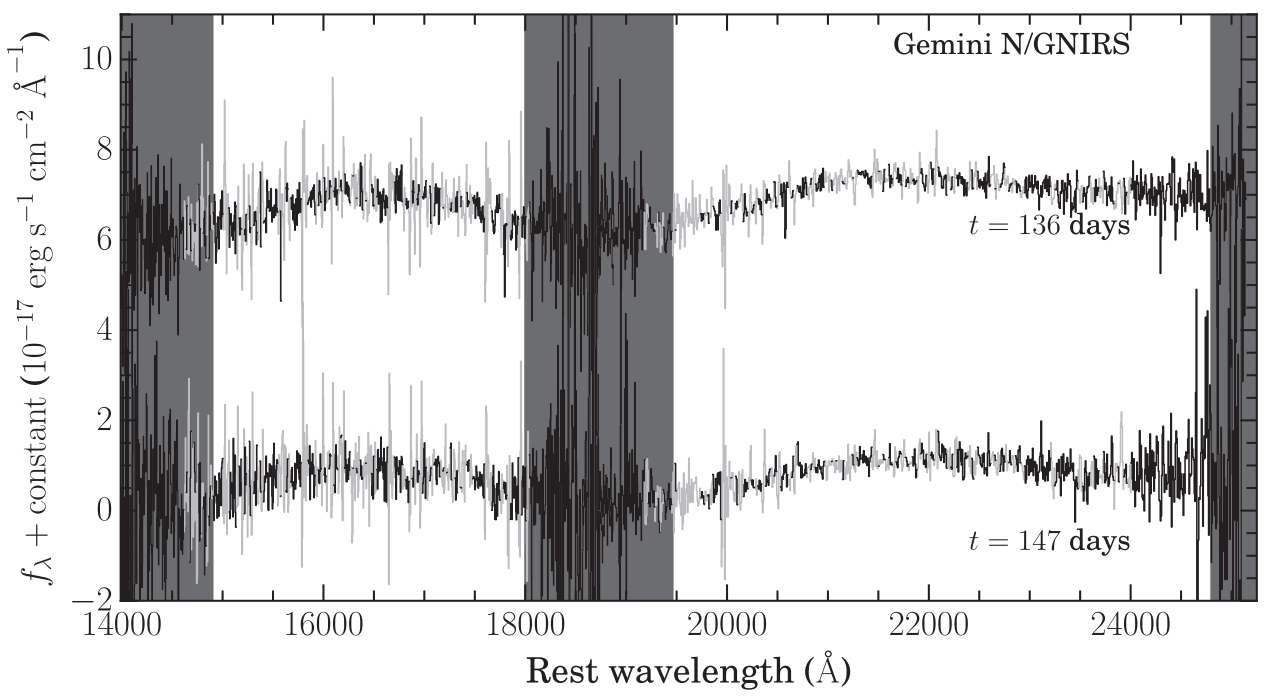

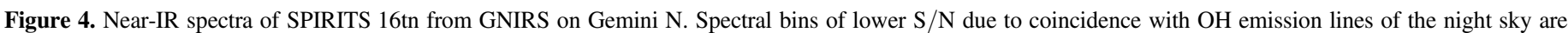

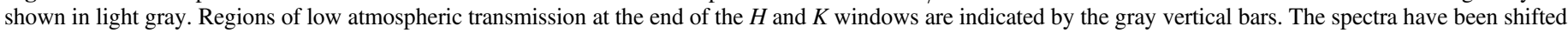

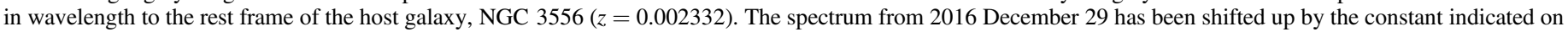
the figure for clarity.

calculated as 5 times the rms noise at the position of the transient in the final radio images.

\section{Analysis}

Here we describe our analysis of both the archival imaging data at the position of the transient and our photometric and spectroscopic measurements of SPIRITS 16tn obtained as part of our follow-up effort.

\subsection{Progenitor Constraints and Host Environment}

We examined the Spitzer/IRAC pre-explosion images of NGC 3556 from 2011 February 7, which we also used as references for image subtraction as described in Section 2.1, for the presence of a possible IR progenitor star. No clear point source is detected in either IRAC channel to $5 \sigma$ limiting magnitudes of [3.6] > $14.6 \mathrm{mag}$ and [4.5] > $14.4 \mathrm{mag}$, where the depth is primarily limited by bright, spatially varying background emission from the host. At the assumed distance to NGC 3556 and correcting only for Galactic extinction, the limits on the absolute magnitudes of the progenitor are $M_{[3.6]}>-15.1 \mathrm{mag}$ and $M_{[4.5]}>-15.3 \mathrm{mag}$.

Images of NGC 3556 were obtained with the HST on 1994 July 4 in program SNAP-5446 (PI: G. Illingworth). These observations used the Wide Field Planetary Camera 2 (WFPC2) with the F606W filter and covered the site of SPIRITS 16tn, approximately $22 \mathrm{yr}$ before its outburst. To determine the precise location of SPIRITS 16tn in the archival WFPC2 F606W image, we registered this frame with the WFC3 F814W detection image of the active transient described above in Section 2.2.1. Using centroid measurements for 10 bright stars detected in both frames, we determined the geometric transformation from WFPC2 to WFC3 using the Space Telescope Science Data Analysis System (STSDAS) ${ }^{21}$ geomap task. By applying the geotran task to the WFPC2 frame and blinking this transformed image against the WFC3 image, we verified the quality of the registration. The rms errors of the geometric fits for the reference stars were 0.15 and

\footnotetext{
${ }^{21}$ STSDAS is a product of STScI, which is operated by AURA for NASA.
}

0.20 pixels in the $x$ and $y$ coordinates, respectively, corresponding to 0.006 and 0.008 arcsec. We did not detect a source consistent with the position of SPIRITS 16tn to a $5 \sigma$ limiting magnitude of $V \gtrsim 24.5 \mathrm{mag}$ in the archival WFPC2 F606W image. We show the location of SPIRITS 16tn in the WFPC2 F606W image in the bottom left panel of Figure 1.

At the distance to NGC 3556 and correcting for only Galactic extinction, this corresponds to an upper limit on the absolute magnitude of the progenitor star of $M_{V} \gtrsim-5.2 \mathrm{mag}$. Assuming a bolometric correction for an intermediate red supergiant (RSG) spectral type M0 of $-1.23 \mathrm{mag}$ from Levesque et al. (2005), and adopting a solar bolometric magnitude of $M_{\odot, \text { bol }}=+4.74 \mathrm{mag}$, we obtain a limit on the luminosity of the progenitor of $L<2.9 \times 10^{4} L_{\odot}$. However, if we assume $A_{V} \approx 7.8 \mathrm{mag}$, as inferred for SPIRITS 16tn in Section 3.3, our limit on the progenitor luminosity becomes far less constraining at $L \lesssim 3.9 \times 10^{7} L_{\odot}$.

We use our PCWI observations to constrain the environment of the progenitor of SPIRITS 16tn. For each pixel in the processed data cube, we fit a simple polynomial to remove the continuum emission from the galaxy. We then measured the $\mathrm{H} \alpha$ flux in each pixel by integrating over the $\mathrm{H} \alpha$ emission line at the known velocity of the galaxy $\left(\approx 699 \mathrm{~km} \mathrm{~s}^{-1}\right)$, to produce a 2D map of $\mathrm{H} \alpha$ flux near the location of the transient (Figure 5). The fluxes were then transformed to an equivalent luminosity using the distance to the galaxy, followed by conversion to an estimated star formation rate using the relations in Kennicutt (1998). The star formation rates in each pixel were then converted to an equivalent star formation surface density using an angular scale of $0.043 \mathrm{kpc} \operatorname{arcsec}^{-1}$. As shown, SPIRITS 16tn is coincident with a dense starforming environment with star formation rate densities of $\sim 10^{-2} M_{\odot} \mathrm{yr}^{-1} \mathrm{kpc}^{-2}$.

\subsection{Light Curves and Color Evolution}

SPIRITS 16tn was discovered at [4.5] $=13.04 \pm 0.05$ mag $\left(M_{[4.5]}=-16.7 \mathrm{mag} ; \lambda L_{\lambda}=1.3 \times 10^{7} L_{\odot}\right)$, as shown in the light curves in Figure 2. The high IR luminosity suggests an explosive event, likely an SN. At [4.5] the flux is observed to fade at a rate 
Table 2

Summary of Radio Observations of SPIRITS 16tn

\begin{tabular}{|c|c|c|c|c|c|c|}
\hline UT Date & MJD & $\begin{array}{l}\text { Phase }^{\mathrm{a}} \\
\text { (days) }^{\text {das }}\end{array}$ & Inst. & $\begin{array}{c}\text { Frequency } \\
(\mathrm{GHz})\end{array}$ & $\begin{array}{l}\begin{array}{l}\text { Flux } \\
(m J y)\end{array} \\
\text { b }\end{array}$ & $\begin{array}{l}\text { Luminosity }{ }^{\mathrm{b}, \mathrm{c}} \\
\left(\mathrm{erg} \mathrm{s}^{-1} \mathrm{~Hz}^{-1}\right)\end{array}$ \\
\hline 2016 Sep 2-5 & $57,633-57,636$ & $17-20$ & AMI-LA & 15.0 & $<0.3$ & $<2.8 \times 10^{25}$ \\
\hline 2016 Sep 4.0 & $57,635.0$ & 19.0 & VLA & 10.0 & $<0.047$ & $<4.4 \times 10^{24}$ \\
\hline 2016 Sep 4.0 & $57,635.0$ & 19.0 & VLA & 3.0 & $<0.10$ & $<9.3 \times 10^{24}$ \\
\hline 2017 Jan 12.4 & $57,765.4$ & 149.4 & VLA & 15.5 & $<0.029$ & $<2.6 \times 10^{24}$ \\
\hline 2017 Jan 12.4 & $57,765.4$ & 149.4 & VLA & 6.0 & $<0.029$ & $<2.7 \times 10^{24}$ \\
\hline
\end{tabular}

Notes.

${ }^{\text {a }}$ Phase is number of days since the earliest detection of this event on 2016 August 15.0 (MJD = 57,615.0).

b $5 \sigma$ limiting magnitudes are given for nondetections.

${ }^{\mathrm{c}}$ Luminosities calculated assuming a distance to NGC 3556 from NED of $8.8 \mathrm{Mpc}$.

of 0.018 mag day $^{-1}$ between $t=0$ and 184.7 days. In the nearIR $K_{s}$ band, SPIRITS $16 \mathrm{tn}$ is observed to fade more slowly at a rate of 0.013 mag day ${ }^{-1}$. These are faster than the expected bolometric decline rates of 0.009 mag day $^{-1}$ for light curves powered by the radioactive decay of ${ }^{56} \mathrm{Co}$ (see, e.g., Gehrz 1988; Gehrz \& Ney 1990). In the $H$ and $J$ bands, the observed decline rates are somewhat slower at $0.009 \pm 0.004 \mathrm{mag} \mathrm{day}^{-1}$ and $0.007 \pm 0.003 \mathrm{mag} \mathrm{day}^{-1}$, respectively.

At discovery, the IR color is [3.6]-[4.5] $=0.676 \pm 0.007$ mag. This corresponds to an effective blackbody temperature of $T_{\text {eff }} \approx 970 \mathrm{~K}$, possibly indicating that emission from warm dust is a significant contributor to the IR luminosity. At 184.7 days, SPIRITS 16tn is observed to have faded more rapidly at [3.6] than at [4.5] and evolves to a redder IR color of [3.6]-[4.5] $\gtrsim 1.0 \mathrm{mag}\left(T_{\text {eff }} \lesssim 700 \mathrm{~K}\right)$.

\subsection{SED}

From our photometry, we constructed quasi-contemporaneous SEDs of SPIRITS 16tn at two epochs. For the first, we adopt the time of the HST/WFC3 detections at $t=41.9$ days as the nominal phase. We use a linear (in magnitudes) interpolation of the [4.5] light curve, and for the $K_{s}$ band we extrapolate the observed decline back from the detection at $t=57.5$ days. We include the earlier Swift/UVOT nondetections as upper limits. As we only have one detection at [3.6] and cannot interpolate an observed decline rate, we consider this point as an upper limit under the assumption that the transient faded in this band between $t=0$ and 41.9 days. For the second epoch, we adopt the time of the second [4.5] detection (and nondetection at [3.6]) at $t=184.7$ days as the nominal phase, using extrapolations of $J$ - and $H$-band decline rates and interpolating the $K_{s}$-band light curve. The photometric magnitudes were converted to band luminosities $\left(\lambda L_{\lambda}\right)$ at the assumed distance to the host and correcting only for Galactic reddening. To convert the UBVI optical points, we adopt Vega flux zero-points and broadband effective wavelengths for the Bessell et al. (1998) Johnson-Cousins-Glass system. We adopt 2MASS system values from Cohen et al. (2003) for our $J H K_{s}$ photometry. For Spitzer [3.6] and [4.5] points, we use the flux zero-points and effective wavelengths listed in the IRAC instrument handbook. We show the SED evolution of SPIRITS 16tn in Figure 6.

\subsubsection{Estimating the Extinction}

The observed SED at $t=41.9$ days is remarkably red. It is likely that SPIRITS 16 tn suffers from a high degree of host extinction given its location along an obvious dust lane in a highly inclined, late-type host galaxy. The photometry of SPIRITS 16tn cannot directly constrain the extinction parameters without some assumptions about the intrinsic SED of the source. We attempt to estimate the extinction to SPIRITS 16 th by comparing the optical/near-IR SEDs to the SEDs of well-studied SNe.

Type II-Plateau SNe (SNe IIP) are the most common of all CCSN subtypes, and we use the IJH light curves of the SN IIP SN 2004et from Maguire et al. (2010) as a template for comparison. Following Maguire et al. (2010), we adopt an explosion date of UT 2004 September 22.0 (MJD $=53,270.0$ ), a distance of $D=5.9 \mathrm{Mpc}$, and a total (Galactic and host) extinction parameterized by $E(B-V)=0.41 \mathrm{mag}$ for SN 2004et. The light curves of SN 2004et are well sampled during each of the canonical phases of SN IIP light-curve evolution: the $\approx 100$-day photospheric plateau phase, the rapid fall-off of the plateau, and the subsequent radioactive decline phase. The absolute phase of SPIRITS 16tn is highly uncertain, as our most constraining pre-explosion upper limit is at 82 days before discovery. Using a linear interpolation of the light curves, we find the host extinction parameter $E(B-V)$ that best reproduces the $I-J$ color of SN 2004et between 50 and 150 days in increments of 1 day. Even across this wide range of possible phases, the color evolution of SN 2004et is such that we find a range of $2.6 \mathrm{mag}<E(B-V)<2.95 \mathrm{mag}$, with a mean value of $E(B-V)=2.8 \mathrm{mag}$.

In the top left panel of Figure 6, we show the observed SED of SPIRITS 16tn at $t=41.9$ days (maximum age of 123.9 days) compared to the SED of SN 2004et at 150 days post explosion, just after the start of radioactive decline phase, but scaled down by a factor of $4.2 \times 10^{-2}$. Applying $E(B-V)=2.7$ mag then provides a good match between the $I-, J-$, and $H$-band measurements to those of SPIRITS 16tn.

We performed a similar analysis using the light curves of SN 2005cs in nearby galaxy M51, a prototypical and wellobserved low-luminosity SN IIP explosion (Pastorello et al. 2006, 2009). With a peak bolometric luminosity of $\approx 6 \times 10^{42} \mathrm{erg} \mathrm{s}^{-1}$, SN 2005cs was $\sim 10$ times fainter than SN 2004et. We adopt a distance to SN 2005cs of $7.1 \mathrm{Mpc}$ (Takáts \& Vinkó 2006), a total foreground extinction (Milky Way and host) of $E(B-V)=0.05$ mag (Baron et al. 2007), 


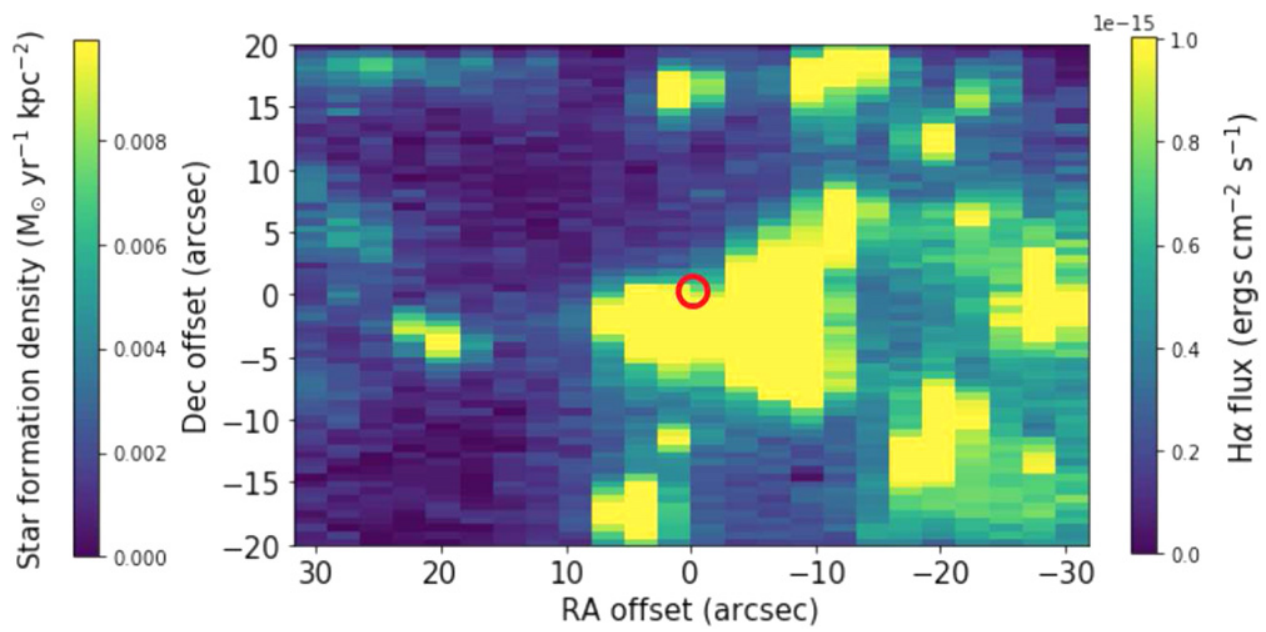

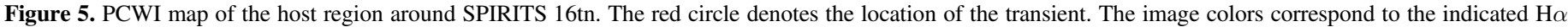
fluxes (right vertical axis) along with their equivalent star formation rate densities (left vertical axis).

and an explosion date of UT 2005 June 27.5 (MJD = 53,548.5) as in Pastorello et al. (2009). While the $I$-band light curve is well sampled throughout the plateau, fall-off, and decline tail, the available near-IR $J$ - and $H$-band photometry is more limited. We compare the SED of SN 2005cs at a phase of 270 days to the late-time SED of SPIRITS 16tn at $t=$ 184.7 days (maximum age of 266.7 days) in the top right panel of Figure 6. We find a suitable match with $E$ $(B-V)=2.5 \mathrm{mag}$ and scaling the SED of SN 2005cs by a factor of 0.76 .

We find that assuming an SN II-like SED for SPIRITS 16tn indicates a high degree of foreground host extinction in the range of $\quad E(B-V) \approx 2.5-3.0 \mathrm{mag} \quad\left(A_{V} \approx 7.8-9.3 \mathrm{mag} \quad\right.$ assuming $R_{V}=3.1$ ), regardless of the absolute phase since explosion. This estimate is high compared to interstellar extinction for typical lines of sight in disk galaxies $\left(A_{V} \approx 1-2 \mathrm{mag} \mathrm{kpc}^{-1}\right)$, but given high inclination of NGC 3556 and the coincidence of SPIRITS 16tn with a clear dust lane, it is not unreasonable. A direct comparison of the luminosity of SPIRITS 16tn is not possible in this analysis because of the large uncertainty in absolute phase. The inferred luminosity of SPIRITS $16 \mathrm{tn}$ at $t=184.7$ days is comparable, however, to a late-phase, low-luminosity, SN 2005cs-like explosion, suggesting that SPIRITS 16tn is likely both heavily obscured and intrinsically faint.

\subsubsection{Blackbody and Dust Component SED Models}

The bright IR emission associated with SPIRITS 16tn likely indicates the presence of warm dust. To model the dust emission, we assume an optically thin distribution of dust of total mass $M_{\mathrm{d}}$, composed of spherical grains of radius $a$, radiating thermally at a single, equilibrium temperature $T_{\mathrm{d}}$. This idealized model is described in more detail by, e.g., Hildebrand (1983), Dwek (1985), and Fox et al. (2010).

The expected flux from the warm dust is

$$
F_{\nu}=M_{\mathrm{d}} \frac{\kappa_{\nu}(a) B_{\nu}\left(T_{\mathrm{d}}\right)}{D^{2}}
$$

where $B_{\nu}\left(T_{\mathrm{d}}\right)$ is the Planck blackbody function, $D$ is the distance from the source, and $\kappa_{\nu}(a)$ is the dust mass absorption coefficient. In what follows, we assume a grain size of $a=0.1 \mu \mathrm{m}$ and use broken power-law approximations to the dust mass absorption coefficients for dust composed entirely of either graphite or silicate (derived from Mie theory; see Figure 4 of Fox et al. 2010). To account for host extinction, we assume $E(B-V)=2.5$ mag based on our comparison with SN 2005cs above. ${ }^{22}$

We fit the IR photometric data for the Spitzer discovery epoch ( $t=0$ days) with this simple dust model for both graphite and silicate compositions to infer $T_{\mathrm{d}}$ and $M_{\mathrm{d}}$. Under the assumption of optically thin dust, the IR luminosity is not sensitive to the size of the dust cloud. In the optically thick case, however, the radius corresponding to blackbody emission provides a lower bound on the dust radius, $R_{\mathrm{d}}$. We repeat the procedure for the quasi-contemporaneous optical-IR SEDs at $t=41.9$ and 187.4 days, including an additional, hotter blackbody component of temperature $T_{*}$ and radius $R_{*}$. We note that for $E(B-V)=2.5$ our optical/near-IR data do not cover the peak of the hotter SED component, and furthermore, the values inferred for $T_{*}$ and $R_{*}$ strongly depend on the choice of extinction. We do not attempt to make strong statements about the properties of the hotter component of the SED for these reasons, and we focus our analysis on the properties of the dust component. The results are shown in the bottom left panel of Figure 6 and summarized in Table 3.

At $t=0$ days, our best-fitting results to the IR SED give $T_{\text {dust }} \approx 680 \quad \mathrm{~K} \quad(880 \mathrm{~K})$ and $M_{\text {dust }} \approx 1.1 \times 10^{-4} M_{\odot}$ $\left(1.5 \times 10^{-4} M_{\odot}\right)$ for graphite (silicate) dust. The blackbody fit to the data gives a higher temperature of $T_{\mathrm{d}} \approx 1100 \mathrm{~K}$ and sets a lower bound on the dust radius of $R_{\mathrm{d}} \gtrsim 4.0 \times 10^{15} \mathrm{~cm}$.

At $t=41.9$ days, we find similar results for the dust temperature for each model, but we infer a somewhat lower mass of $M_{\text {dust }} \approx 0.4 \times 10^{-4} M_{\odot}\left(0.7 \times 10^{-4} M_{\odot}\right)$ for graphite (silicate) dust and a smaller bound on the dust radius from the blackbody fit of $R_{\mathrm{d}} \gtrsim 2.5 \times 10^{15} \mathrm{~cm}$. We note, however, that the uncertainties in interpolating the [4.5] and $K_{s}$-band light curves to construct the SED $t=41.9$ days may have artificially lowered these values.

At $t=187.4$ days, the data are no longer well fit by a hot source component and warm dust. The best-fitting results find $T_{\mathrm{d}} \approx 700-1000 \mathrm{~K}$ but are notably inconsistent with the upper limit at [3.6]. The blackbody dust radius is smaller by a factor

\footnotetext{
${ }^{22}$ Here we use a (Cardelli et al. 1989) extinction $R_{V}=3.1$ to deredden the photometry to match our analysis using the dust radiative code DUSTY in Section 3.3.3.
} 


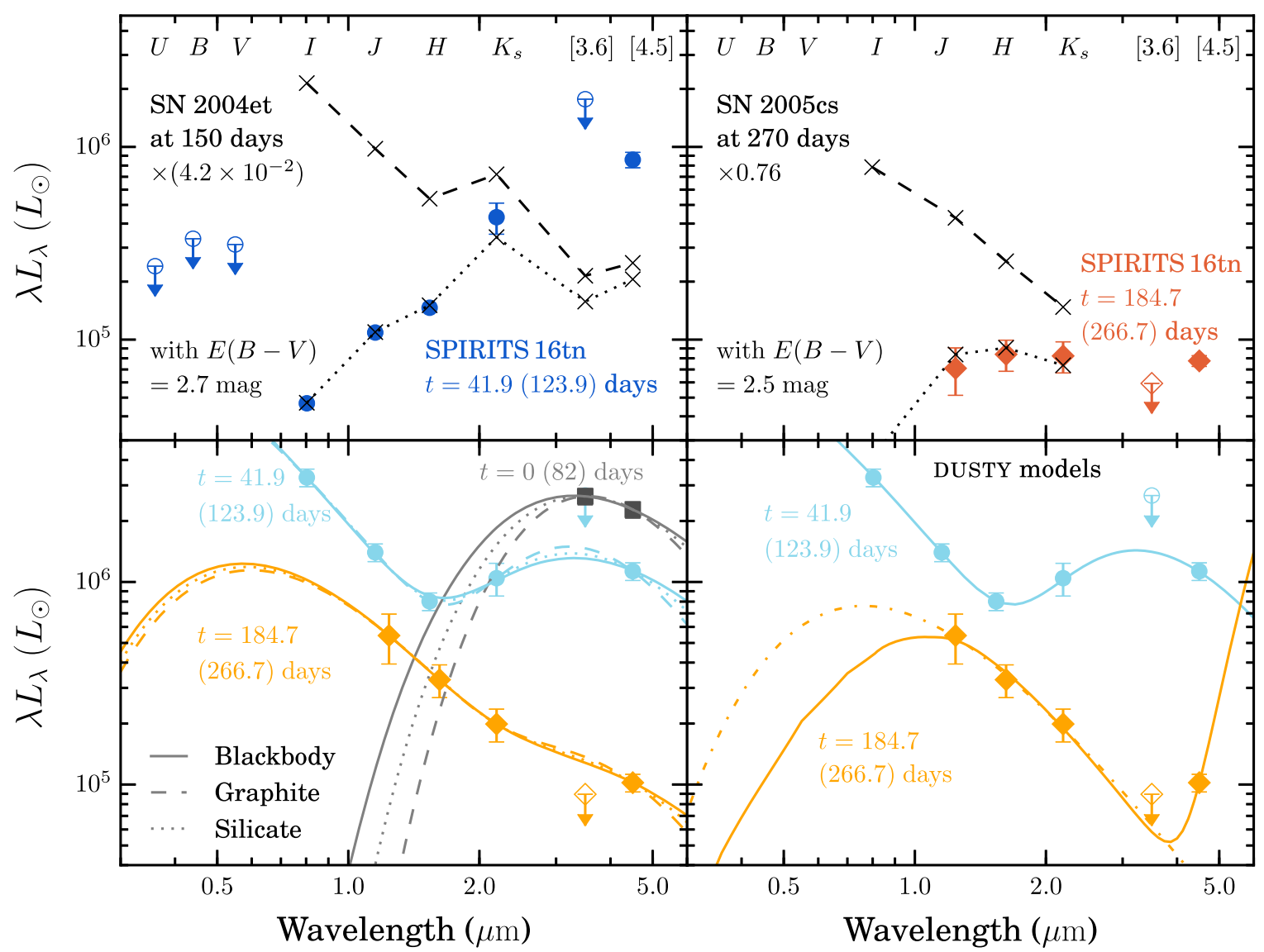

Figure 6. Top panels: SED evolution of SPIRITS 16tn, corrected only for Galactic extinction, constructed from the available broadband photometry at nominal phases of $t=41.9$ days (maximum age of 123.9 days; dark-blue circles) and $t=184.7$ days (maximum age of 266.7 days; dark-orange diamonds). Open symbols with downward-pointing arrows indicate points treated as upper limits. We compare the observed SED at $t=41.9$ days to that of SN 2004et at a phase of 150 days as the black crosses and dashed curve, scaled down in luminosity by a factor of $4.2 \times 10^{-2}$. The black crosses and dotted curve show this SED reddened by $E(B-V)=$ 2.7 mag. Similarly, we compare the observed SED of SPIRITS $16 \mathrm{tn}$ at $t=187.4$ days to that of the low-luminosity SN $2005 \mathrm{cs}$ at a phase of 270 days scaled down by a factor of 0.76 (dashed black curve) and reddened by $E(B-V)=2.5$ mag (dashed black curve). Bottom panels: SEDs of SPIRITS 16tn corrected for an assumed reddening of $E(B-V)=2.5 \mathrm{mag}$ at the discovery epoch (gray squares), $t=41.9$ days (light-blue circles), and $t=184.7$ days (light-orange diamonds). In the bottom left panel, we show multicomponent fits to the SEDs using blackbodies (solid curves) and optically thin warm dust components with opacities appropriate for graphite (dashed curves) and silicates (dotted curves). In the bottom right panel, we show best fits to the SEDs from our dust radiative transfer modeling using DUSTY as the light-blue and light-orange solid curves. We also show an additional model for the photometry at $t=184.7$ days as the dashed orange curve, treating the [4.5] flux as an upper limit, as it is likely enhanced by $\mathrm{CO}$ emission at this phase.

of 3-5 compared to the earlier epochs, and the inferred dust masses are lower by at least a factor of 10. As discussed below in the context of a CCSN, the flux at [4.5] at this phase is likely enhanced by emission from the fundamental vibrational transition of $\mathrm{CO}$ and is not attributable solely to thermal emission from warm dust. The lack of evidence for a warm dust component at this phase may indicate that the dust has cooled, shifting the flux to longer wavelengths not probed by our data. Alternatively, as discussed below in Section 4.2.2, the early presence and subsequent disappearance of the warm dust component may be interpreted as evidence for an IR echo, i.e., the reprocessing of the $\mathrm{UV} /$ optical emission from the luminosity peak of the transient into the thermal IR by a shell of preexisting circumstellar dust.

\subsubsection{SED Modeling with DUSTY}

In addition to the simple dust models described above, we modeled the SED of SPIRITS 16tn using the dust radiative transfer code DUSTY (Ivezic \& Elitzur 1997; Ivezic et al. 1999; Elitzur \& Ivezić 2001). To find best-fitting models and allowed parameter ranges, we use a Markov chain Monte Carlo (MCMC) wrapper around DUSTY. Here we use a spherically symmetric distribution of graphite dust from Draine \& Lee (1984) with a standard MRN grain size distribution $\left(d n / d a \propto a^{-3.5}, 0.005<a<0.25 \mu \mathrm{m}\right.$; Mathis et al. 1977). For the central luminosity source, we assume a simple blackbody spectrum and allow the model to find best-fitting values for the source temperature $T_{*}$ and luminosity $L_{*}$, the optical depth in $V$ band due to circumstellar dust $\tau_{V}$, the dust temperature $T_{\mathrm{d}}$, and the inner dust radius $R_{\mathrm{d}}$. The source temperature and luminosity depend strongly on the assumed foreground extinction, but for simplicity, we again fix the extinction at $E(B-V)=2.5 \mathrm{mag}$ as above. Our implementation of DUSTY uses a Cardelli et al. (1989) extinction law with $R_{V}=3.1$, but the differences from the Fitzpatrick (1999) law assumed throughout the majority of this work are small at the wavelengths of interest in the optical and near-IR.

In this model, the dust is heated by the central source, i.e., the inferred properties of the dust are not independent of $L_{*}$ and $R_{*}$. As $T_{\mathrm{d}}$ is constrained strongly by the shape of the IR SED, 
Table 3

Results of Blackbody and Dust Component SED Modeling

\begin{tabular}{|c|c|c|c|c|c|c|}
\hline $\begin{array}{l}\text { Phase }^{a} \\
\text { (days) }\end{array}$ & $\begin{array}{l}T_{*} \\
(\mathrm{~K})\end{array}$ & $\log R_{*} / \mathrm{cm}$ & $\begin{array}{c}T_{\mathrm{d}} \\
(\mathrm{K})\end{array}$ & $\log R_{\mathrm{d}} / \mathrm{cm}$ & $\begin{array}{c}M_{\mathrm{d}} \\
\left(10^{-4} M_{\odot}\right)\end{array}$ & Dust Type \\
\hline \multirow[t]{2}{*}{0.0} & $\ldots$ & $\ldots$ & 1100 & 15.6 & $\ldots$ & blackbody \\
\hline & $\ldots$ & $\ldots$ & 680 & $\ldots$ & 1.1 & graphite \\
\hline \multirow[t]{3}{*}{41.9} & 17,000 & 13.7 & 1100 & 15.4 & $\cdots$ & blackbody \\
\hline & 14,000 & 13.7 & 730 & $\ldots$ & 0.4 & graphite \\
\hline & 15,000 & 13.7 & 900 & $\ldots$ & 0.7 & silicate \\
\hline & 6300 & 13.9 & 810 & $\ldots$ & 0.07 & silicate \\
\hline
\end{tabular}

Note.

${ }^{\text {a }}$ Phase is number of days since the earliest detection of this event on 2016 August 15.0 (MJD = 57,615.0).

Table 4

Results of SED Modeling with DUSTY ${ }^{\mathrm{a}}$

\begin{tabular}{|c|c|c|c|c|c|c|c|c|c|c|}
\hline $\begin{array}{l}\text { Phase }^{\mathrm{b}} \\
\text { (days) }\end{array}$ & \multicolumn{2}{|c|}{$\log L_{*} / L_{\odot}$} & \multicolumn{2}{|c|}{$T_{*}$} & \multicolumn{2}{|c|}{$\tau_{V}$} & \multicolumn{2}{|c|}{$\frac{T_{\mathrm{d}}}{(\mathrm{K})}$} & \multicolumn{2}{|c|}{$\log R_{\mathrm{d}} / \mathrm{cm}$} \\
\hline 41.9 & 7.4 & $7.1_{-0.2}^{+0.3}$ & 15200 & $10900_{-2700}^{+4500}$ & 0.06 & $0.2_{-0.1}^{+0.2}$ & 840 & $810_{-90}^{+80}$ & 16.8 & $16.7_{-0.2}^{+0.2}$ \\
\hline $187.4^{\mathrm{c}}$ & 6.0 & $6.4_{-0.7}^{+1.6}$ & 48100 & $7600_{-4100}^{+23800}$ & $\ldots$ & $\ldots$ & $\ldots$ & $\ldots$ & $\ldots$ & $\ldots$ \\
\hline
\end{tabular}

Notes.

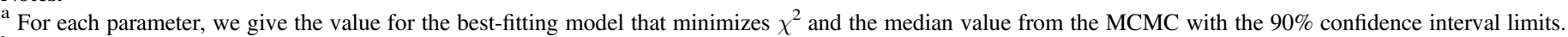

${ }^{\mathrm{b}}$ Phase is number of days since the earliest detection of this event on 2016 August 15.0 (MJD = 57,615.0).

${ }^{\mathrm{c}}$ Results for $t=187.4$ days when [4.5] flux is treated as an upper limit including only a single blackbody component.

we do not expect it to vary strongly with the other model parameters. Furthermore, it is fairly robust to the choice of extinction, as the effects of reddening are small in the IR. We expect, however, $R_{\mathrm{d}}$ and $\tau_{V}$ to vary strongly with the central source properties, namely, a hotter, more luminous central source will force dust at a given temperature to larger radii and correspondingly lower optical depths. This model also does not account for light-travel time effects inherent to dust at large radii from an evolving, transient source.

The results of our DUSTY modeling at both epochs are given in Table 4, including both the values for each parameter for the best-fitting model, i.e., the model that minimizes $\chi^{2}$, and the median value and $90 \%$ confidence interval limits from the MCMC posterior distributions. We note that the best-fitting values are sometimes near the extrema of the posterior distributions. The best-fitting SEDs are also shown in the bottom right panel of Figure 6 in comparison to the observations.

At $t=41.9$ days, there is a clear IR excess requiring a warm dust component in addition to the interior, hotter source component. The MCMC results for this model have a dust temperature of $T_{\mathrm{d}}=810_{-90}^{+80} \mathrm{~K}$, consistent with the best-fitting value from our simple, optically thin graphite dust model at this epoch in Section 3.3.2, and an inner dust radius of $R_{\mathrm{d}}=5.0_{-1.9}^{+2.9} \times 10^{16} \mathrm{~cm}$ at $90 \%$ confidence. As expected, $T_{\mathrm{d}}$ is well constrained by the $K_{s}$-band and [4.5] fluxes and does not vary strongly with the other parameters of the model. While producing a significant IR excess, the dust is optically thin at $\tau_{V}=0.2_{-0.1}^{+0.2}$. Because our photometry does not cover the peak of the hot blackbody component, our measurements can only place a lower limit on the temperature and luminosity of the source, and the upper confidence limits found by the MCMC are not physically meaningful. We infer $T_{*} \gtrsim 8200 \mathrm{~K}$ and $L_{*} \gtrsim 7.9 \times 10^{6} L_{\odot}$, but note that these limits are highly dependent on our choice of foreground host extinction, i.e., for $E(B-V)_{\text {host }}<2.5 \mathrm{mag}$, a lower blackbody temperature and luminosity would be consistent with the data.

At $t=187.4$ days, the hot component has faded by a factor of $\approx 2.7$ in the $J$ and $H$ bands. Again, our photometric measurements do not cover the peak of this component, and thus the results of the MCMC modeling only allow us to estimate a lower limit on the source temperature of $T_{*}>3200 \mathrm{~K}$. The flux in the redder bands, however, has faded more quickly. The DUSTY model fits a dust component to the excess flux at [4.5] with constraints from the MCMC at $T_{\mathrm{d}}=370_{-170}^{+400} \mathrm{~K}$, but there is a strong degeneracy between the cooler dust temperatures at smaller radii $(\approx 6 \times$ $\left.10^{15} \mathrm{~cm}\right)$ and warmer dust at large radii $\left(\approx 5 \times 10^{19} \mathrm{~cm}\right)$. Again, it is likely that the flux at [4.5] is enhanced by emission from the fundamental vibrational mode of $\mathrm{CO}$, and therefore it is probably not attributable to thermal emission from dust. Treating the measurement at [4.5] instead as an upper limit, we find that the SED of SPIRITS 16tn at $t=187.4$ days can be adequately modeled with a single blackbody component with $T_{*}>4500 \mathrm{~K}$ and $L_{*}>5.0 \times 10^{5} L_{\odot}$.

\subsection{The Optical and Near-IR Spectra}

At $t=79$ days after first detection, the optical spectrum of SPIRITS 16tn is characterized by a faint, red continuum. There are no clearly discernible features. The apparent dip in the spectrum near $9300 \AA$ is coincident with a strong telluric absorption band and is probably not intrinsic to the source. 
The near-IR spectra of SPIRITS 16tn are shown in Figure 4. Though the Gemini N/GNIRS spectra covered the entire nearIR spectral range from 8500 to $25000 \AA$, we detect emission from SPIRITS 16tn only in the $H$ and $K$ regions of the spectrum. Due to uncertainty in the age of SPIRITS 16tn at discovery, the phase of SPIRITS 16tn is only constrained to be between 136 and 229 days since explosion at the time the spectra were taken.

As in the optical spectrum, we detect a red continuum associated with SPIRITS 16tn, but there are no unambiguous features in the near-IR. Though the spectra appear to peak near the centers of the $H$ and $K$ spectral windows, we suspect that this may be an artifact of low $\mathrm{S} / \mathrm{N}$ and poor flux calibration, particularly near the edges of the bands, where little flux is received through the atmosphere. As expected given the high degree of reddening inferred from the SED, we detect the strongest continuum emission in the $K$ spectral region, with an overall decrease in flux toward the blue.

\section{Discussion}

Here we compare the observed properties of SPIRITS 16tn to those of various $\mathrm{SNe}$ subtypes to inform our interpretation of the observations.

\subsection{Comparison to SNe Ia}

The deep radio nondetections of SPIRITS 16tn (Section 2.4) may be easily explained if it is an SN Ia. No SN Ia has been detected as a radio source to deep limits in radio luminosity as far down as $L_{\nu} \lesssim 10^{24} \mathrm{erg} \mathrm{s}^{-1} \mathrm{~Hz}^{-1}$ for the nearest events (e.g., Panagia et al. 2006; Chomiuk et al. 2016). To test the hypothesis that SPIRITS 16tn is an SN Ia, we compare its IR color evolution and near-IR spectrum to well-studied events.

In the top right panel of Figure 7, we show the [4.5] light curve of SPIRITS 16tn compared to several SNe Ia from Johansson et al. (2017), who found that SNe Ia form a homogeneous class of objects at these wavelengths. The phases of the SPIRITS 16tn observations are shown as days since maximum, with $t=0$ assumed to be at the time of the Spitzer discovery observations and where the uncertainty in the time of maximum light is indicated by the horizontal error bars. SPIRITS 16tn shows a similar decline in luminosity at [4.5] to the sample of SNe Ia, but the [3.6]-[4.5] color evolution, shown in the bottom right panel of Figure 7, is notably inconsistent. At discovery, SPIRITS 16tn had a very red [3.6][4.5] color of $0.7 \mathrm{mag}$ and evolved to an even redder color of [3.6]-[4.5] $>1.0$ mag over a period of 185 days. In contrast, $\mathrm{SNe}$ Ia, which may be somewhat red at early times, evolve quickly to the blue, reaching [3.6]-[4.5] $\approx-1 \mathrm{mag}$ at a phase of $\approx 150$ days. SNe Ia may evolve again to redder colors at very late times, but the observed color of SPIRITS 16tn is too extreme for $\mathrm{SNe}$ Ia across the entire range of phases relevant here.

Redder [3.6]-[4.5] colors have been observed during the first 400 days in some thermonuclear $\mathrm{SNe}$, e.g., the interactionpowered SN Ia-CSM SN 2005gj (Fox \& Filippenko 2013) and the unusual, dusty SN Iax SN 2014dt (Fox et al. 2016). We show these $\mathrm{SNe}$ as the gray symbols in the top right panel of Figure 7. Both events have an observed late-time, IR flux excess at [4.5] over a normal SN Ia light curve extending past 200 days, indicative of emission from warm dust. In the context of SN Ia light curves, SPIRITS 16tn does not show such a late-time excess despite its red color.

We have not considered the effects of extinction from the host galaxy or local environment of the $\mathrm{SN}$, e.g., from circumstellar dust. To produce a color excess of 0.7 mag between [3.6] and [4.5] would require an additional $A_{V} \gtrsim 40$ mag of extinction, using the empirically derived broadband extinction parameters for [3.6] and [4.5] from Chapman et al. (2009), much higher than the inferred extinction to SPIRITS 16tn from the SED of $A_{V} \approx 8 \mathrm{mag}$.

Furthermore, the featureless spectrum of SPIRITS 16tn is wholly inconsistent with normal SNe Ia at comparable phases. In Figure 8 , we show the $1.48-1.9 \mu \mathrm{m}$ region $(\approx H$ band $)$ of the spectrum of SPIRITS 16tn, along with the late-time spectra of SN Ia SN 2014J at a phase of 128 days (post $B$-band maximum) from Johansson et al. (2017) and the SN Ia SN 2005df at 198, 217, and 380 days (Diamond et al. 2015). The phase of our near-IR spectrum is constrained to be between 136 and 229 days. The late-time spectra of SNe Ia are dominated by blended, nebular emission features, primarily forbidden transitions of Fe-peak elements (Bowers et al. 1997; Spyromilio et al. 2004). In the $H$-band region, Diamond et al. (2015) specifically identified transitions of [Fe II], [Co II], and [Co III] in the spectra of SN Ia SN 2005df, as labeled in Figure 8 . They noted that the spectrum became Fe dominated as the Co features faded between $\approx 200$ and 380 days; however, the strong, broad emission lines of [Fe II] persisted to very late phases. These features are completely absent from the near-IR spectrum of SPIRITS 16tn, and thus we definitively rule out a reddened SN Ia in this case.

\subsection{Comparison to CCSNe}

We compare the light curve at [4.5] of SPIRITS 16tn to CCSNe of Type II and stripped-envelope Types IIb and Ib/c in the top left and middle panels of Figure 7. Among the hydrogen-rich SNe II, we do not distinguish between the photometric subtypes IIP and IIL, defined by the presence of a light-curve plateau or linear decline, respectively. Our data set for SPIRITS 16tn is insufficient to make such a distinction. Furthermore, the existence of the two truly distinct subclasses is debated, and recent studies of large SN II samples have suggested that SNe IIP and SNe IIL may instead form a continuous distribution in their observed properties (e.g., Anderson et al. 2014; Sanders et al. 2015; Rubin \& Gal-Yam 2016).

The observed [4.5] luminosity peak of SPIRITS 16tn at 16.7 mag is in the range of SNe II but is $\gtrsim 1$ mag fainter than is observed for the sample of stripped-envelope events. Notably, SPIRITS 16tn fades more rapidly at [4.5] at $0.017 \mathrm{mag}^{-1}{ }^{-1}$ than any of the CCSNe for which comparable data were available. The fastest event in the comparison sample is the SN II SN 2013ej (black octagons in the top left panel of Figure 7) fading at a rate of 0.013 mag day ${ }^{-1}$. Although the sample of stripped-envelope $\mathrm{SNe}$ is small, they appear relatively more homogeneous at [4.5] compared to SNe II, with typical decline rates between 0.009 and $0.012 \mathrm{mag} \mathrm{day}^{-1}$. Given the larger degree of variation in both peak luminosity at [4.5] and the observed decline rate for SNe II, it is easier to reconcile the lower [4.5] peak and faster decline of SPIRITS 16tn with the sample of SNe II.

As shown in the bottom panels of Figure 7, SPIRITS 16tn develops a very red color by $t=184.7$ days of [3.6]-[4.5] $>1.0 \mathrm{mag}$. 


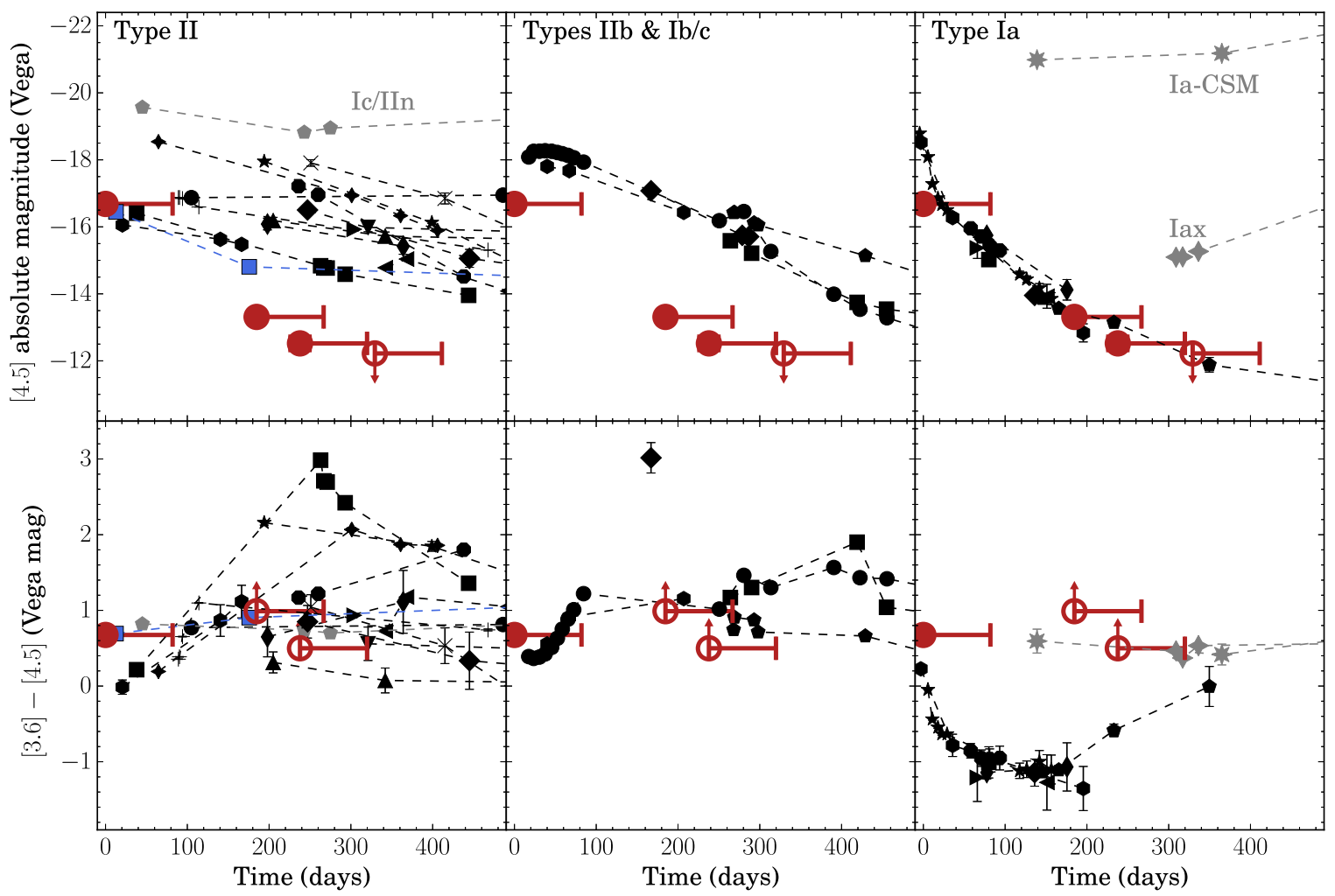

Figure 7. [4.5] light curves (top row) and [3.6]-[4.5] color evolution (bottom) for SPIRITS 16tn (red circles) compared to SNe II (left column), stripped-envelope $\mathrm{SNe} \mathrm{IIb}$ and $\mathrm{Ib} / \mathrm{c}$ (middle column), and thermonuclear SNe Ia (right column) shown in black. Lower limits in color are indicated by open circles and upward-pointing arrows. Time is given on the $x$-axes as days since discovery for the core-collapse events and days since $B$-band maximum for SNe Ia. The uncertainty in the phase for SPIRITS 16tn is indicated by the red horizontal error bars. The sample of SNe IIP shown in the right column includes the Spitzer/IRAC measurements for SN 2004A (thick diamonds), SN 2005ad (thin diamonds), SN 2005af (stars), SN 2006my (upward-pointing triangles), SN 2006ov (downward-pointing triangles), and SN 2007oc (crosses) from Szalai \& Vinkó (2013) and references therein; SN 2011ja (circles), SN 2013am (leftward-pointing triangles), SN 2013bu (rightwardpointing triangles), SN 2013ej (octagons), and SN 2014bi (squares) from Tinyanont et al. (2016) and references therein; SN 2004dj (plus signs; Kotak et al. 2005; Meikle et al. 2011; Szalai et al. 2011); SN 2004et (four-point stars; Kotak et al. 2009); and SPIRITS 14buu (hexagons; Jencson et al. 2017). Also shown is the interaction-powered SN Ic/IIn SN 2014C (Tinyanont et al. 2016, and references therein) as the gray pentagons and the luminous infrared transient SN 2008S (Adams et al. 2016b) as the blue squares. The stripped-envelope SNe shown in the middle column include SN 2011dh (circles; Type IIb), SN 2013df (squares; Type IIb), SN 2013dk (pentagons; Type Ic), and SN 2014L (hexagons, Type Ic) from Tinyanont et al. (2016) and references therein, as well as the more recent event SPIRITS 15C (diamonds; Type Ib or IIb; Jencson et al. 2017). Measurements for SNe Ia from Johansson et al. (2017) and references therein are shown in the right column for SN 2005df (thin diamonds), SN 2006X (thick diamonds), SN 2007af (leftward-pointing triangles), SN 2007le (rightward-pointing triangles), SN 2007 sr (squares), SN 2009ig (hexagons), SN 2011fe (pentagons), SN 2012cg (octagons), and SN 2014J (stars). We also show the unusual SN Iax SN 2014dt (Fox et al. 2016, and references therein) and the interaction-powered SN Ia-CSM SN 2005gj (Fox \& Filippenko 2013) as gray four- and eight-pointed stars, respectively. Color measurements for each object are corrected only for Galactic extinction to their respective hosts from NED. Error bars are sometimes smaller than the plotting symbols.

Similarly red colors have been observed at comparable phases for several CCSNe, including the SN IIP SN 2005af (Szalai \& Vinkó 2013, and references therein), SN 2004et (Kotak et al. 2009), and SN 2014bi (Tinyanont et al. 2016), and for the SN IIb/ Ib event SPIRITS 15C (Jencson et al. 2017). While a red mid-IR color may be a signature of thermal emission from warm dust $\left(T_{\text {eff }} \lesssim 700 \mathrm{~K}\right.$ for [3.6]-[4.5] $>1.0 \mathrm{mag}$ ), emission from the $1-0$ vibrational transition of $\mathrm{CO}$ at $\approx 4.65 \mu \mathrm{m}$ can produce excess flux at [4.5] compared to the other mid-IR bands. This emission feature has been directly identified in the mid-IR spectra of several SNe II, including SN 1987A (e.g., Meikle et al. 1989; Wooden et al. 1993), SN 2004dj (Kotak et al. 2005), and SN 2005af (Kotak et al. 2006). Corroborating the identification of this feature, the band heads of the $\Delta \nu=2$ vibrational overtones of $\mathrm{CO}$, which produce excess emission beyond $2.3 \mu \mathrm{m}$ at the end of the $K$ band, have also been observed in, e.g., SN 1987A (Meikle et al. 1989), SN 2004dj (Kotak et al. 2005), and the stripped-envelope events SN 2011dh (Ergon et al. 2015) and SPIRITS 15C (Jencson et al. 2017). We do not clearly detect this feature in our near-IR spectra from $t=136$ to
145 days, but note that the $\Delta \nu=2$ vibrational overtones may be significantly weaker than the fundamental band at [4.5] and hidden in our low-S/N spectra. Furthermore, the spectra were obtained at an earlier epoch, possibly before $\mathrm{CO}$ formed in the ejecta. In the context of CCSNe, we consider CO emission to be the most likely explanation for the observed mid-IR color evolution of SPIRITS 16tn, indicating the presence of $\mathrm{CO}$ in the ejecta by $t \approx 185$ days.

In Figure 2, we compare the multiband light curves of SPIRITS 16tn to those of the low-luminosity SN IIP SN 2005cs. The light curves of SN 2005cs are shifted to the distance of SPIRITS 16tn and reddened with $E(B-V)=2.5$ mag, as inferred from our SED comparison in Section 3.3. For the relative phase offset shown, and with an additional offset in apparent magnitude of $\Delta m=0.7 \mathrm{mag}$ (factor of $\approx 2$ in flux), the late-time $I J H K_{s}$ light curves can be reasonably well matched to those of SPIRITS 16tn. In this scenario, our HST observations of SPIRITS 16tn at $t=41.9$ days would have occurred just after the transition to the nebular phase and 


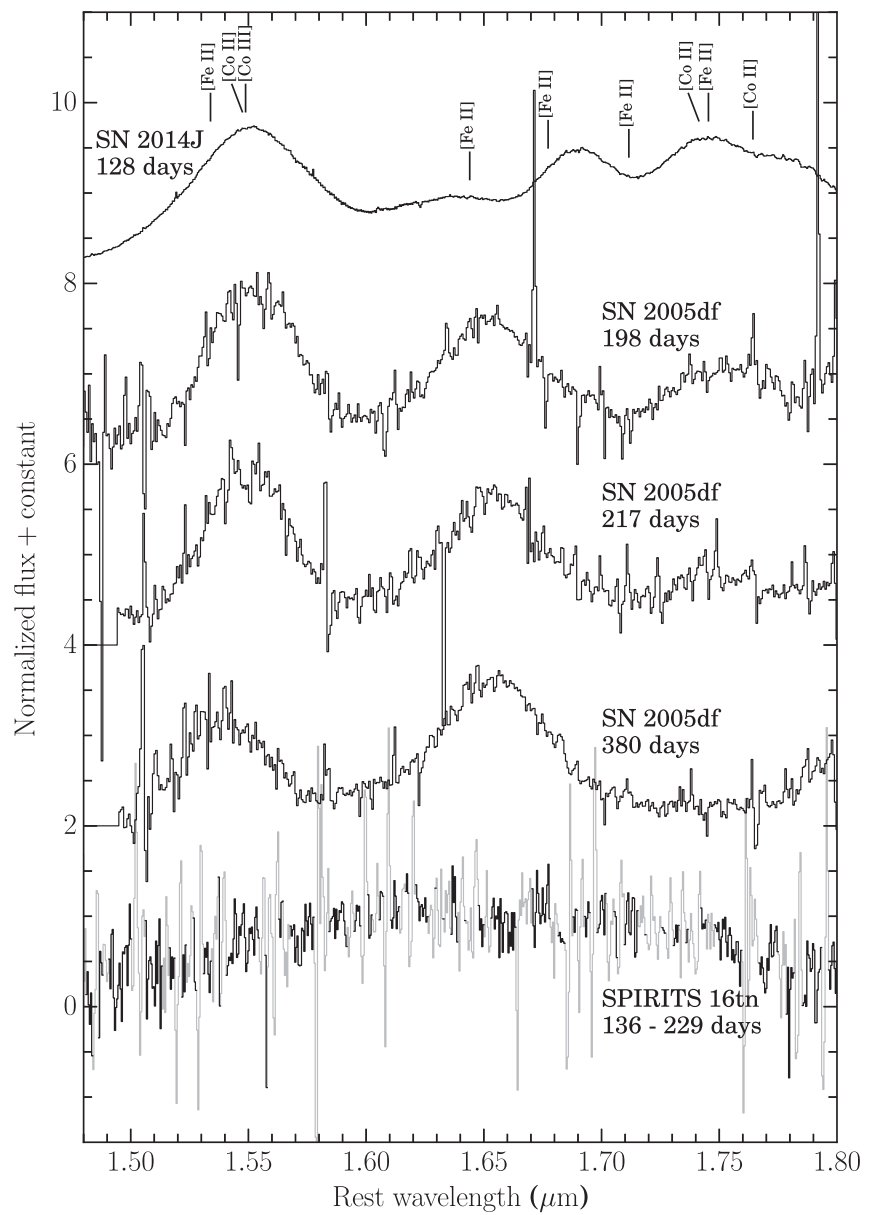

Figure 8. $H$-band spectrum of SPIRITS $16 \mathrm{tn}$, at a phase between 136 and 229 days, compared to late-time, nebular spectra of SNe Ia SN 2014J (Johansson et al. 2017) and SN 2005df (Diamond et al. 2015). The SPIRITS 16tn spectrum is the average of the two Gemini N/GNIRS spectra taken on 2016 December 29 and 2017 January 9. Spectral bins of lower S/N due to coincidence with an $\mathrm{OH}$ emission line of the night sky are shown in light gray. The spectra have been normalized by the flux at $1.644 \mu \mathrm{m}$, and each spectrum is shifted up from the one below for clarity. Forbidden transitions of Fe-peak elements identified in Diamond et al. (2015) are indicated above the spectrum of SN 2014J. These broad, blended features are not present in SPIRITS 16tn.

require a plateau duration $\lesssim 123.9$ days to be consistent with our $z$-band pre-explosion nondetection. Given our lack of earlytime data for SPIRITS 16tn and the notable gap in near-IR photometric coverage of SN 2005cs during the transition to the nebular phase, we cannot perform a more detailed light-curve comparison. Still, we find the optical-near-IR light curve evolution of SPIRITS 16tn to be largely consistent with an SN 2005cs-like, low-luminosity Type IIP event.

In Figure 9, we compare near-IR spectra of SPIRITS 16tn at a phase between 136 and 229 days post maximum to those of CCSNe of various types, including the Type Ic broad-lined (Ic-BL) SN 1998bw (Patat et al. 2001), the Type IIn SN 2010j1 (Borish et al. 2015), the Type IIb SN 2011dh (Ergon et al. 2015), and the Type II SN 2013ej (Yuan et al. 2016). Some of the most prominent features identified in late-phase CCSNe in the $H$ and $K$ bands are labeled in Figure 9, including $\mathrm{Mg} \mathrm{I}$ at $1.504 \mu \mathrm{m}$, blended [Fe II] at $1.644 \mu \mathrm{m}$ and [Si I] at $1.646 \mu \mathrm{m}$, $\mathrm{He}$ I at $2.058 \mu \mathrm{m}, \mathrm{Br} \gamma$ at $2.166 \mu \mathrm{m}$, and the band heads of the $\Delta \nu=2$ vibrational overtones of $\mathrm{CO}$ beyond $2.3 \mu \mathrm{m}$. $\mathrm{Pa} \alpha$, a

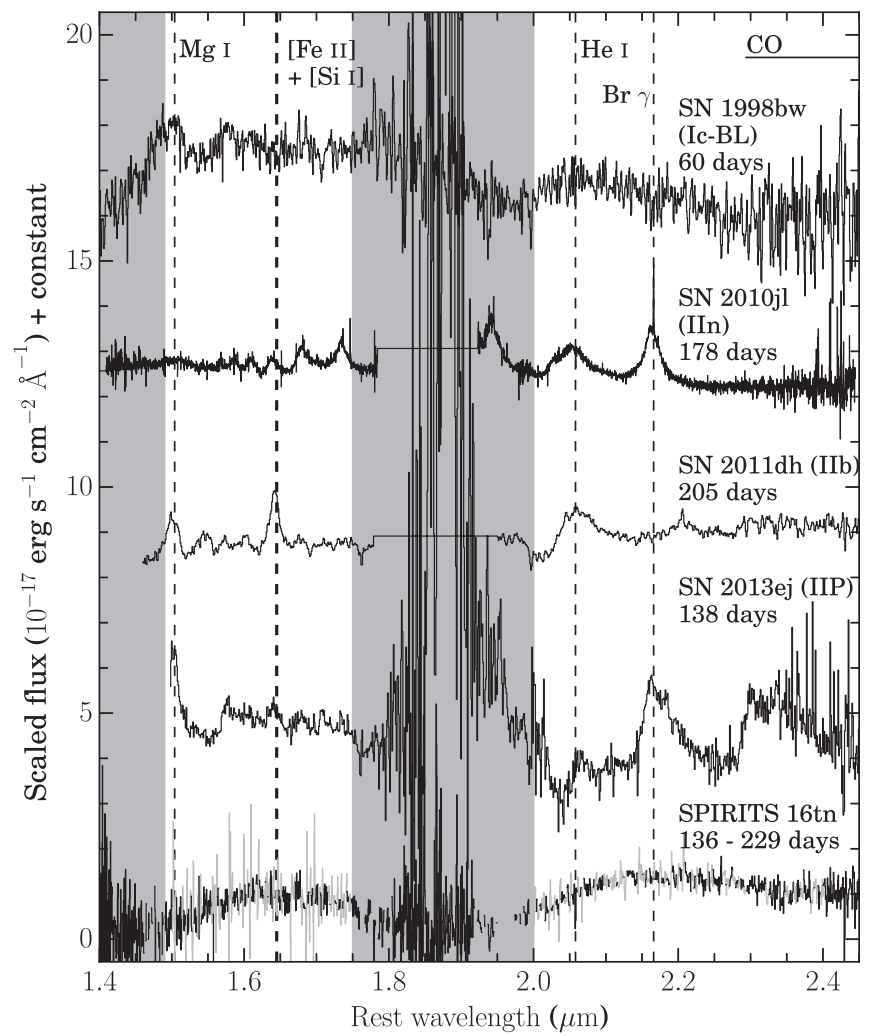

Figure 9. $H$ - and $K$-band spectra of SPIRITS 16 th at a phase between 136 and 229 days, along with the late-phase spectra of CCSNe of various types for comparison. The CCSN spectra are scaled in flux to the distance of SPIRITS 16tn and reddened by $E(B-V)=2.5$ mag. Each one is shifted up from the one below by an arbitrary constant for clarity. Prominent features present in the spectra of some CCSNe are indicated by the dashed vertical lines and labeled near the top of the figure.

typically strong $\mathrm{H}$ I feature in $\mathrm{SNe}$ II, is unfortunately in the low atmospheric transmission region between the $H$ and $K$ spectral windows, where we did not receive any detectable flux from SPIRITS 16tn.

As we do not detect any clear features in SPIRITS 16tn, we are unable to provide a definitive classification. However, the lack of a clear spectroscopic signature of the interaction of the SN ejecta with a dense CSM, often observed as superimposed narrow $\left(\sim\right.$ few $\left.\times 100 \mathrm{~km} \mathrm{~s}^{-1}\right)$ and broad $\left(\sim\right.$ few $\left.\times 1000 \mathrm{~km} \mathrm{~s}^{-1}\right)$ components of the $\mathrm{H}$ I and He I features, can rule out a strongly interacting SN IIn. We suggest that at late times it is possible that the near-IR spectral features of noninteracting CCSNe may be very weak.

\subsubsection{Radio Limits}

In Figure 10, we show our limits on the radio luminosity of SPIRITS 16th as a function of phase compared to the peak radio luminosities and times to peak for CCSNe. Radio emission is produced in CCSNe when the fastest SN ejecta interact with and shock the slow-moving pre-explosion CSM from the pre-explosion stellar wind of the progenitor. As the shock wave propagates through the CSM, turbulent instabilities amplify magnetic fields and accelerate relativistic electrons (Chevalier 1982). The resultant radio emission is characterized by slowly declining, optically thin, nonthermal synchrotron and early, optically thick absorption at low frequencies. Proposed 


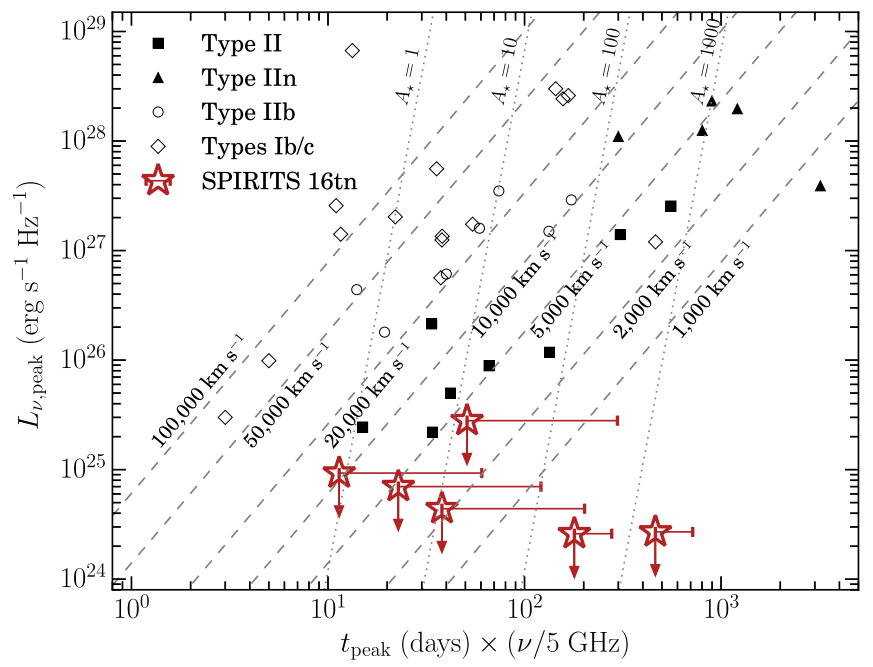

Figure 10. Peak radio luminosity vs. time of peak times the frequency of observation for radio CCSNe adapted from, e.g., Chevalier et al. (2006) and Romero-Cañizales et al. (2014). SNe II are shown as black squares, and interaction-powered SNe IIn are shown as black triangles. Open circles and diamonds represent stripped-envelope $\mathrm{SNe} \mathrm{IIb}$ and $\mathrm{Ib} / \mathrm{c}$, respectively. Upper limits on the radio luminosity of SPIRITS 16tn at a given phase from our nondetections are shown as red open stars with downward-pointing arrows, where the horizontal error bars represent our uncertainty in the absolute phase since explosion. Assuming an SSA model with an electron distribution with $p=3$ for the shock wave propagating through the CSM, one can infer the shock velocity (dashed lines) and CSM density parameter $\left(A_{\star} ;\right.$ dotted lines) from the position on this diagram.

absorption mechanisms include synchrotron self-absorption (SSA) or internal free-free absorption in the emitting region and free-free absorption by the external, ionized CSM (e.g., Chevalier 1982, 1998).

If one assumes that SSA is dominant, for an electron population with an energy spectral index of $p=3$, the size of the radio-emitting region at the time of the SSA peak can be calculated as (Chevalier 1998)

$$
\begin{aligned}
R_{\mathrm{s}}= & 4.0 \times 10^{14} \alpha^{-1 / 19}\left(\frac{f}{0.5}\right)^{-1 / 19}\left(\frac{F_{\mathrm{p}}}{\mathrm{mJy}}\right)^{9 / 19} \\
& \times\left(\frac{D}{\mathrm{Mpc}}\right)^{18 / 19}\left(\frac{\nu}{5 \mathrm{GHz}}\right)^{-1} \mathrm{~cm},
\end{aligned}
$$

where $\alpha \equiv \epsilon_{e} / \epsilon_{B}$ is the ratio of the energy density in relativistic electrons to that in the magnetic field, $f$ is the fraction of the spherical volume filled by the radio-emitting region, $F_{p}$ is the peak flux at frequency $\nu$, and $D$ is the distance to the source. If additional absorption mechanisms are important, this radius must be even larger. The shock wave velocities, $v_{\mathrm{s}}$, inferred for $\alpha=1$ (assuming equipartition) and $f=0.5$ (as estimated in Chevalier \& Fransson 2006) are shown as the dashed lines in Figure 10. We the note weak dependence in Equation (2) of the shock radius on these parameters.

For a steady, pre-SN stellar wind, the density profile of the $\mathrm{CSM}$ as a function of radius, $r$, is $\rho_{\mathrm{w}}=A / r^{2} \equiv \dot{M} /\left(4 \pi r^{2} v_{\mathrm{w}}\right)$, where $\dot{M}$ is the mass-loss rate and $v_{\mathrm{w}}$ is the wind velocity. $A \equiv \dot{M} /\left(4 \pi v_{\mathrm{w}}\right)$ is the normalization of the CSM density profile as in Chevalier (1982). As calculated by Chevalier \& Fransson (2006), the radio emission at time $t$ since explosion of a CCSN is sensitive to the density profile of the CSM as

$$
\begin{aligned}
& A_{\star} \epsilon_{B-1} \alpha^{8 / 19}=1.0\left(\frac{f}{0.5}\right)^{-8 / 19}\left(\frac{F_{\mathrm{p}}}{\mathrm{mJy}}\right)^{-4 / 19} \\
& \times\left(\frac{D}{\mathrm{Mpc}}\right)^{-8 / 19}\left(\frac{\nu}{5 \mathrm{GHz}}\right)^{2}\left(\frac{t}{10 \text { days }}\right)^{2},
\end{aligned}
$$

where $\epsilon_{B-1} \equiv \epsilon_{B} / 0.1$ and $A_{\star} \equiv A /\left(5 \times 10^{11} \mathrm{~g} \mathrm{~cm}^{-1}\right)$ is a dimensionless proxy for $A$. We show lines of constant $A_{\star}$ in Figure 10, determined largely by the strong dependence of this parameter on $t_{\text {peak }}$.

Our deep nondetections of SPIRITS 16tn indicate either that this event is an intrinsically weak radio source or that the emission is heavily absorbed. Though we do not rule out radio emission arising at very late times, characteristic of strongly interacting SNe IIn with a dense CSM, this scenario is unlikely given the lack of prominent interaction features in the optical/ near-IR spectra. Our observations are inconsistent with most varieties of stripped-envelope events, which tend to be more luminous radio sources. A high-velocity $\left(v_{\mathrm{s}} \gtrsim 50,000 \mathrm{~km} \mathrm{~s}^{-1}\right)$ SN Ic with a fast-evolving radio light curve, however, is not explicitly ruled out (cf. SN 2007gr, Soderberg et al. 2010; and PTF12gzk, Horesh et al. 2013a). Our optical-IR SED analysis and comparisons with well-studied SNe II indicate that SPIRITS 16tn falls at the low end of the SN luminosity function, and a weak SN II radio counterpart is consistent with our observations. Using Equation (3), for typical SN II shock velocities of $v_{\mathrm{s}} \approx$ few $\times 10^{3} \mathrm{~km} \mathrm{~s}^{-1}$, our nondetections can constrain $A_{\star} \epsilon_{B-1} \alpha^{8 / 19} \lesssim 24$. For a steady pre-SN wind, we then infer a limit on the pre-SN mass-loss rate of $\dot{M} \lesssim 2.4 \times 10^{-6}\left(\frac{\epsilon_{B}}{0.1}\right)^{-1} \alpha^{-8 / 19}\left(\frac{v_{\mathrm{w}}}{10 \mathrm{~km} \mathrm{~s}^{-1}}\right) M_{\odot} \mathrm{yr}^{-1}$.

Such mass-loss rate is consistent with a low-luminosity RSG $\left(L \approx 10^{4.5}-10^{5} L_{\odot}\right)$ based on the standard observational prescriptions of de Jager et al. (1988; see, e.g., Figure 3 of Smith 2014). This supports the picture of SPIRITS 16tn as a low-luminosity SN II arising from the explosion of a low-mass $\left(M \approx 10-15 M_{\odot}\right)$ RSG progenitor. We note that, depending on the assumed explosion date of SPIRITS 16tn, for $v_{\mathrm{s}}=10,000 \mathrm{~km} \mathrm{~s}^{-1}$ and $v_{\mathrm{w}}=10 \mathrm{~km} \mathrm{~s}^{-1}$, the timing of our radio observations probes the mass-loss history of the progenitor only in the final $\approx 50-600 \mathrm{yr}$ before the explosion.

Concurrent panchromatic observations spanning the radio to the X-ray have indicated deviations from energy equipartition in some SNe. For example, for SN 2011dh Soderberg et al. (2012) find $\alpha=30$ and $\epsilon_{B}=0.01$. Adopting such values results in only a modest decrease in the shock velocity (using Equation (2)) by a factor of 1.2 and an increase in our limit on the mass-loss rate by a factor of 2.4. Alternatively for SN 2011dh, Horesh et al. (2013b) find $\alpha \approx 500-1700$, adopting a value of 1000 as a reasonable average, and $\epsilon_{B}=3 \times 10^{-4}$. This still results in only a modest decrease in the inferred shock velocity by a factor of 1.4 , and for a fixed wind velocity, the limit on the mass-loss rate increases by a factor of 20 .

\subsubsection{Origin of the Observed Dust Component}

In Section 3.3, our modeling of the SED of SPIRITS 16th at $t=0$ and 41.9 days suggests the presence of an IR component $\left(T_{\mathrm{d}} \approx 700-900 \mathrm{~K}\right)$ powered by thermal emission by at least $M_{\mathrm{d}} \approx(1.0-1.5) \times 10^{-4} M_{\odot}$ of warm dust (our observations are not sensitive to any additional dust at cooler temperatures). 
By $t=187.4$ days, we no longer see evidence for this warm dust component, indicating that either it has faded or the dust has cooled, shifting the flux to longer wavelengths.

The dust emission may arise from either preexisting circumstellar dust formed in the pre-SN wind of the progenitor or newly formed dust in the dense, rapidly cooling ejecta behind the SN shock. For a shock velocity of $v_{s}=10,000$ $\mathrm{km} \mathrm{s}^{-1}$, the radius of the shock at a phase of 82.0 days (the maximum age of SPIRITS 16tn at $t=0$ days) is $R_{s}=$ $7.1 \times 10^{15}\left(v_{s} / 10^{4} \mathrm{~km} \mathrm{~s}^{-1}\right) \mathrm{cm}$. As we infer a lower limit on the dust radius (blackbody radius from Section 3.3.2) of $R_{\mathrm{d}} \gtrsim 4.0 \times 10^{15} \mathrm{~cm}$ that is smaller than the shock radius, it is plausible that the emitting dust is located in the post-shock cooling zone. Given the low observed dust temperatures, however, it is unlikely that the early dust component is due to newly formed dust in the ejecta. We would expect newly formed dust to be near the evaporation temperature, as, given sufficiently high densities, dust grains will begin to condense as soon as the drop in temperature of the radiation environment allows. Typical values for astrophysical dust are $T_{\text {evap }} \approx 1900$ $\mathrm{K}$ for graphite and $T_{\text {evap }} \approx 1500 \mathrm{~K}$ for silicate, significantly hotter than the observed dust component.

For preexisting dust, we can interpret the observed IR excess as an IR echo. In this scenario, a preexisting shell of dust is heated by the peak luminosity of the explosion and reradiates this energy thermally in the IR. The duration of the echo is related to the size of the dust shell from geometrical arguments as $\Delta t \sim 2 R_{\mathrm{d}} / c$. The observations of the warm dust component between $t=0$ and 41.9 days, and subsequent fading by $t=187.4$ days, when the maximum age of SPIRITS $16 \mathrm{tn}$ is 269.4 days, would then require $5.4 \times 10^{16} \mathrm{~cm} \lesssim R_{\mathrm{d}} \lesssim 3$. $5 \times 10^{17} \mathrm{~cm}$.

As a consistency check, we can estimate the peak luminosity, $L_{\text {peak }}$, of the transient required to heat spherical dust grains of radius $a$ within this range of distances to the observed temperatures. The energy absorbed by a dust grain is balanced by the energy it radiates as

$$
\frac{L_{\mathrm{peak}}}{4 \pi R_{\mathrm{d}}^{2}} \pi a^{2} Q_{\mathrm{abs}}=4 \pi a^{2} \sigma_{\mathrm{SB}} T_{\mathrm{d}}^{4} Q_{\mathrm{em}}
$$

The peak luminosity of the transient is then given by

$$
L_{\text {peak }}=16 \pi R_{\mathrm{d}}^{2} \sigma_{\mathrm{SB}} T_{\mathrm{d}}^{4} \frac{Q_{\mathrm{em}}}{Q_{\mathrm{abs}}},
$$

where $Q_{\mathrm{em}}$ and $Q_{\mathrm{abs}}$ are Planck-averaged emission and absorption efficiencies for Laor \& Draine (1993) dust grains. For the temperature of the incident radiation field we assume values $T_{\text {rad }}=10,000 \mathrm{~K}$ and $T_{\text {rad }}=6000 \mathrm{~K}$, characteristic of an $\mathrm{SN}$ at peak. We use a value of $\sigma_{\mathrm{SB}}=5.67 \times 10^{-5}$ erg $\mathrm{cm}^{-2} \mathrm{~K}^{-4} \mathrm{~s}^{-1}$ for the Stefan-Boltzmann constant. For graphite grains of size $a=0.1 \mu \mathrm{m}$ at $T_{\mathrm{d}}=680 \mathrm{~K}$, we find $4.7 \times 10^{40} \mathrm{erg} \mathrm{s}^{-1} \lesssim L_{\text {peak }} \lesssim 1.9 \times 10^{42} \mathrm{erg} \mathrm{s}^{-1}$ for $T_{\text {rad }}=$ $10,000 \mathrm{~K}$ and similar values of $5.7 \times 10^{40} \mathrm{erg} \mathrm{s}^{-1} \lesssim L_{\text {peak }} \lesssim$ $2.3 \times 10^{42} \mathrm{erg} \mathrm{s}^{-1}$ for $T_{\text {rad }}=6000 \mathrm{~K}$. Alternatively, for grains of silicate composition at $T_{\mathrm{d}}=880 \mathrm{~K}$, we find a somewhat higher values of $9.9 \times 10^{41} \mathrm{erg} \mathrm{s}^{-1} \lesssim L_{\text {peak }} \lesssim 4.0 \times 10^{43} \mathrm{erg} \mathrm{s}^{-1}$ for $T_{\text {rad }}=10,000 \mathrm{~K}$, or $2.6 \times 10^{41} \mathrm{erg} \mathrm{s}^{-1} \lesssim L_{\text {peak }} \lesssim 1.0 \times$ $10^{43} \mathrm{erg} \mathrm{s}^{-1}$ for $T_{\text {rad }}=6000 \mathrm{~K}$.

While these estimates are crude, we can still compare them to the observed range of peak luminosities for SNe II. Faran et al. (2018), for example, estimate the bolometric luminosities of $29 \mathrm{SNe}$ II and find peak values spanning at least two orders of magnitude and ranging from $L_{\mathrm{bol}} \approx 2.4 \times 10^{41} \mathrm{erg} \mathrm{s}^{-1}$ to $2.4 \times 10^{43} \mathrm{erg} \mathrm{s}^{-1}$. Similarly, the pseudo-bolometric (from $\sim U$ to $I$ band) light curves of the sample of $\mathrm{SNe}$ II from Valenti et al. (2016) span $L_{\text {bol }} \approx 1.0 \times 10^{41} \mathrm{erg} \mathrm{s}^{-1}$ to $5 \times$ $10^{42} \mathrm{erg} \mathrm{s}^{-1}$. Among the faintest $\mathrm{SNe}$ II known, the quasibolometric UBVRI light curve of SN 1999br peaked at only $4.5 \times 10^{40} \mathrm{erg} \mathrm{s}^{-1}$ (Pastorello et al. 2004, 2009). The observed range of peak luminosities for $\mathrm{SNe}$ II is similar to the range of luminosities estimated above to explain the IR excess of SPIRITS 16tn as a dust echo.

We can also estimate the pre-SN mass-loss rate of the progenitor star necessary to support such an echo. We assume that the dust is concentrated in a thin shell with $\Delta r / R_{\mathrm{d}}=0.1$, a dust-to-gas ratio of $M_{\mathrm{d}} / M_{\mathrm{g}}=0.01$, and again a pre-SN wind velocity of $v_{\mathrm{w}}=10 \mathrm{~km} \mathrm{~s}^{-9}$ and find $\dot{M} \approx 9 \times 10^{-6}-6 \times 10^{-5}$ $\left(\frac{M_{\mathrm{d}}}{10^{-4} M_{\odot}}\right)\left(\frac{M_{\mathrm{d}} / M_{\mathrm{g}}}{0.01}\right)^{-1}\left(\frac{\Delta r / R_{\mathrm{d}}}{0.1}\right)^{-1}\left(\frac{v_{\mathrm{w}}}{10 \mathrm{~km} \mathrm{~s}^{-1}}\right) M_{\odot} \mathrm{yr}^{-1}$ for the range of $R_{\mathrm{d}}$ allowed by the observations. These estimates are a factor of $\approx 4-25$ higher than that in Section 4.2.1 based on the radio observations, but they probe an earlier time in the mass-loss history of the progenitor of somewhere between $\approx 1700$ and $11,000 \mathrm{yr}$ before explosion. While such mass-loss rates would imply a more luminous and massive RSG progenitor $\left(L \approx 10^{5.2}-10^{5.5} L_{\odot}\right.$, again assuming standard de Jager et al. 1988 prescriptions), if the dust is confined to a shell, this could indicate a relatively brief episode of enhanced mass loss.

\subsection{Non-SN Transient Scenarios}

As we are unable to provide a definitive spectroscopic classification of SPIRITS 16tn as a CCSN, we briefly consider other observed classes of luminous IR transients as possible explanations for this event.

\subsubsection{SN 2008 S and NGC 300 OT2008-1-like Transients}

SN 2008S and the luminous 2008 optical transient in NGC 300 (NGC 300 OT2008-1) are the prototypes of a distinct class of transients. They have optically obscured progenitors but bright mid-IR pre-explosion counterparts $\left(M_{[4.5]}<-10 \mathrm{mag}\right)$, suggested to be extreme asymptotic giant branch stars of intermediate mass $\left(\approx 10-15 M_{\odot}\right)$ self-obscured by a dense, dusty wind (Prieto et al. 2008; Bond et al. 2009; Thompson et al. 2009). They are less luminous than typical CCSNe at peak $\left(L_{\mathrm{bol}} \approx 10^{41} \mathrm{erg} \mathrm{s}^{-1}\right.$ for SN 2008S; Botticella et al. 2009). Emission lines in their spectra indicate slow expansion velocities of 70-80 $\mathrm{km} \mathrm{s}^{-1}$ (e.g., Bond et al. 2009; Humphreys et al. 2011). A proposed physical scenario is a weak explosion, possibly an electron-capture $\mathrm{SN}$, or massive stellar eruption that destroys most of the obscuring dust, allowing the transient to be optically luminous. The development of a late-time IR excess, however, suggests that the dust reforms, obscuring the optical transient at late times (e.g., Thompson et al. 2009; Kochanek 2011; Szczygiełet al. 2012). Both events are now fainter than their progenitor luminosities at [3.6] and [4.5], suggesting that the transients were terminal events (Adams et al. 2016b).

The [4.5] light curve of SN 2008S is shown as the blue squares in Figure 7 compared to SPIRITS 16tn and several SNe II. The peak luminosity at [4.5] is similar to SPIRITS 16tn. Furthermore, the peak bolometric luminosity of SN 2008S-like events is sufficient to power the IR dust echo discussed in Section 4.2.2. The IR luminosity of SPIRITS 16tn 
declines more rapidly, and we do not observe a late-time IR excess powered by newly formed dust, inconsistent with the characteristic evolution of SN 2008S-like events.

\subsubsection{Massive Stellar Mergers}

The 2011 transient in NGC 4490 (hereafter NGC 4490-OT; Smith et al. 2016) and M101 2015OT-1 (Blagorodnova et al. 2017) are proposed massive analogs of the galactic contact binary merger V1309 Sco (Tylenda et al. 2011) and the B-type stellar merger V838 Mon (Bond et al. 2003; Sparks et al. 2008). These events typically have unobscured, optical progenitors; irregular, multipeaked light curves, increasingly red colors with time; and significant late-time IR excesses powered by copious dust formation. Their spectra show relatively narrow emission features of $\mathrm{H} \mathrm{I}$ indicating low velocities of $\sim 100 \mathrm{~km} \mathrm{~s}^{-1}$.

In the IR, NGC 4490-OT peaked at $M_{[4.5]} \approx-15 \mathrm{mag}, \approx$ 1.7 mag fainter than SPIRITS 16tn. The inferred mass for the progenitor system of NGC $4490-\mathrm{OT}$ was $20-30 M_{\odot}$, and thus a merger origin for the more luminous SPIRITS 16tn would likely require an exceptionally massive progenitor. Furthermore, the IR light curve of NGC 4490-OT is long-lived, remaining too luminous at phases $\gtrsim 800$ days to be powered by an IR echo (Smith et al. 2016). The relatively short-lived IR excess of SPIRITS 16tn, interpreted here as an IR echo, is generally inconsistent with the observed IR evolution typical of massive star mergers.

\subsubsection{SN Impostors}

Given the low inferred luminosity and lack of radio detection for SPIRITS 16tn, an "SN impostor" may also be a plausible explanation for the observed characteristics of SPIRITS 16tn. SN impostors are nonterminal, massive star outbursts (typically an $M \gtrsim 20-25 M_{\odot}$ luminous blue variable [LBV]) that mimics the appearance of a true SN (e.g., Smith et al. 2011; Van Dyk $\&$ Matheson 2012). These events are are typically fainter in the optical fainter than true $\mathrm{SNe}\left(M_{V} \lesssim-14 \mathrm{mag}\right)$. Impostors have in some cases been mistakenly classified as SNe IIn, due to the presence of "narrow" hydrogen emission lines on top of an "intermediate-width" base $\left(v \sim 1500-2000 \mathrm{~km} \mathrm{~s}^{-1}\right)$. Some impostor outbursts have been observed to directly precede the death of the progenitor in the final years before the SN explosion, e.g., SN 2006jc (Pastorello et al. 2007) and SN 2009ip (Mauerhan et al. 2013; Pastorello et al. 2013). An impostor may be distinguished from a terminal SN explosion by the identification of a surviving progenitor star, or the detection of subsequent outbursts, also indicating that the progenitor has survived.

We detect no clear spectroscopic signatures of an SN or impostor in SPIRITS 16tn and see no evidence of a subsequent outburst or variability in $1.5 \mathrm{yr}$ of continued monitoring with Spitzer post discovery. We also cannot directly test for the survival of the progenitor with currently available data. Deep, high-resolution, near-IR imaging may be able to constrain the presence of a surviving star after the light from the transient completely fades.

\section{Summary and Conclusions}

SPIRITS 16tn is a luminous $\left(M_{[4.5]}=-16.7 \mathrm{mag}\right)$ mid-IR transient discovered with Spitzer/IRAC during the ongoing
SPIRITS survey in the nearby galaxy NGC 3556 . We believe that SPIRITS 16tn is a possible SN. Despite being one of the nearest $\mathrm{SNe}$ discovered in 2016 at only $8.8 \mathrm{Mpc}$, it was completely missed by optical searches owing to heavy extinction. The transient position is coincident with a dark dust lane in the inclined, star-forming disk of the host. We estimate a total extinction of $A_{V}=7.8-9.3 \mathrm{mag}$, making SPIRITS 16tn one of the most highly obscured SNe yet discovered in the IR.

The [4.5] light curve shows a fast decline of 0.018 mag day $^{-1}$, and the source becomes increasingly red in the mid-IR from [3.6]-[4.5] $=0.7 \mathrm{mag}$ to $\gtrsim 1.0$ mag between $t=0$ and 184.7 days post discovery. The optical and near-IR spectra display a featureless, red continuum, ruling out an SN Ia, but preclude a definitive spectroscopic classification. The SED at $t=41.9$ days post discovery is extremely red and can be matched to an SN II-like SED with $E(B-V)=$ 2.5-3.0 mag of extinction. Furthermore, our analysis suggests that SPIRITS 16tn may be an intrinsically dim event similar to the well-studied, low-luminosity SN 2005cs. Modeling of the SED indicates the presence of a warm dust component $(T \approx 700-900 \mathrm{~K})$, which fades by $t=184.7$ days. This is consistent with an IR echo powered by a circumstellar shell of dust located somewhere between $5.4 \times 10^{16} \mathrm{~cm}$ and $3.5 \times 10^{17} \mathrm{~cm}$ heated by a peak luminosity of $\sim 5 \times 10^{40} \mathrm{erg}$ $\mathrm{s}^{-1}$ to $4 \times 10^{43} \mathrm{erg} \mathrm{s}^{-1}$, similar to the range of observed peak luminosities for SNe II.

The source is not detected to deep limits in the radio across frequencies of 3-15.5 GHz, constraining the radio luminosity to $\lesssim 4 \times 10^{24} \mathrm{erg} \mathrm{s}^{-1} \mathrm{~Hz}^{-1}$ between $t=19$ and 149.4 days. This effectively rules out most stripped-envelope $\mathrm{SNe}$, except possibly the most rapidly evolving, high-velocity events that may peak in the radio at very early times. A late-rising, interaction-powered SN IIn may be consistent with our radio limits, but the typically strong spectroscopic signatures of interaction with a dense CSM are absent from our optical/nearIR spectra. SNe II, typically the weakest radio emitters among $\mathrm{CCSNe}$, are the most consistent with our deep radio limits, and in this context we can constrain the pre-SN mass-loss rate of the progenitor to $\dot{M} \lesssim 2.4 \times 10^{-6}\left(\frac{\epsilon_{B}}{0.1}\right)^{-1}\left(\frac{v_{\mathrm{w}}}{10 \mathrm{~km} \mathrm{~s}^{-1}}\right) M_{\odot} \mathrm{yr}^{-1}$. This is consistent with a lower-mass RSG progenitor of $M \sim 10-15 M_{\odot}$.

We analyzed the available pre-explosion Spitzer/IRAC 2011 imaging and HST/WFPC2 F606W imaging of NGC 3556 covering the site of SPIRITS 16tn from 1994, and we do not detect a candidate progenitor star. Given the high degree of extinction inferred to SPIRITS 16tn, however, we are unable to place meaningful limits on the progenitor luminosity.

Taken together, we find the most likely explanation for the observed properties of SPIRITS 16tn to be an SN II explosion that both is highly obscured by foreground, host-galaxy dust and is intrinsically low luminosity. This discovery strengthens the fact that, even in the local $10 \mathrm{Mpc}$ volume, SN searches appear to be incomplete. Transient surveys in the IR have the unique ability to find dust-obscured or otherwise optically dim events, allowing for the true nearby SN population to be uncovered.

We thank R. Lunnan for executing our Keck/LRIS observations. We thank D. Neill and M. Matuszewski for assistance with the PCWI observations and data reduction. We thank A. Monson 
for help with P200/WIRC data reduction. We thank T. Cantwell, Y. Perrott, and the AMI staff for conducting the AMI-LA observations and data reduction. We also thank the anonymous referee for suggestions that improved the paper.

This material is based on work supported by the National Science Foundation Graduate Research Fellowship under grant no. DGE-1144469. H.E.B. acknowledges support for this work provided by NASA through grants GO-13935 and GO-14258 from the Space Telescope Science Institute, which is operated by AURA, Inc., under NASA contract NAS 5-26555. R.D.G. was supported in part by the United States Air Force. A.H. acknowledges support by the I-Core Program of the Planning and Budgeting Committee and the Israel Science Foundation.

This work is based in part on observations made with the Spitzer Space Telescope, which is operated by the Jet Propulsion Laboratory, California Institute of Technology, under a contract with NASA. The work is based, in part, on observations made with the Nordic Optical Telescope, operated by the Nordic Optical Telescope Scientific Association at the Observatorio del Roque de los Muchachos, La Palma, Spain, of the Instituto de Astrofisica de Canarias.

This work is based in part on observations with the NASA/ ESA Hubble Space Telescope obtained at the Space Telescope Science Institute and from the Mikulski Archive for Space Telescopes at STScI, which are operated by the Association of Universities for Research in Astronomy, Inc., under NASA contract NAS5-26555. These observations are associated with programs GO-14258 and SNAP-5446.

Some of the data presented herein were obtained at the W. M. Keck Observatory, which is operated as a scientific partnership among the California Institute of Technology, the University of California, and the National Aeronautics and Space Administration. The Observatory was made possible by the generous financial support of the W. M. Keck Foundation. The authors wish to recognize and acknowledge the very significant cultural role and reverence that the summit of Maunakea has always had within the indigenous Hawaiian community. We are most fortunate to have the opportunity to conduct observations from this mountain.

RATIR is a collaboration between the University of California, the Universidad Nacional Autonóma de México, NASA Goddard Space Flight Center, and Arizona State University, benefiting from the loan of an H2RG detector from Teledyne Scientific and Imaging. RATIR, the automation of the Harold L. Johnson Telescope of the Observatorio Astronómico Nacional on Sierra San Pedro Maŕtir, and the operation of both are funded by the partner institutions and through NASA grants NNX09AH71G, NNX09AT02G, NNX10AI27G, and NNX12AE66G; CONACyT grant INFR2009-01-122785; UNAM PAPIIT grant IN113810; and a UC MEXUS-CONACyT grant.

UKIRT is owned by the University of Hawaii (UH) and operated by the UH Institute for Astronomy; operations are enabled through the cooperation of the East Asian Observatory. When the data reported here were acquired, UKIRT was supported by NASA and operated under an agreement among the University of Hawaii, the University of Arizona, and Lockheed Martin Advanced Technology Center; operations were enabled through the cooperation of the East Asian Observatory.

Based on observations obtained at the Gemini Observatory acquired through the Gemini Observatory Archive and processed using the Gemini IRAF package, which is operated by the Association of Universities for Research in Astronomy, Inc., under a cooperative agreement with the NSF on behalf of the Gemini partnership: the National Science Foundation (United States), the National Research Council (Canada), CONICYT (Chile), Ministerio de Ciencia, Tecnología e Innovación Productiva (Argentina), and Ministério da Ciência, Tecnologia e Inovação (Brazil).

The National Radio Astronomy Observatory is a facility of the National Science Foundation operated under cooperative agreement by Associated Universities, Inc.

The Legacy Surveys consist of three individual and complementary projects: the Dark Energy Camera Legacy Survey (DECaLS; NOAO Proposal ID No. 2014B-0404; PIs: David Schlegel and Arjun Dey), the Beijing-Arizona Sky Survey (BASS; NOAO Proposal ID No. 2015A-0801; PIs: Zhou Xu and Xiaohui Fan), and the Mayall z-band Legacy Survey (MzLS; NOAO Proposal ID No. 2016A-0453; PI: Arjun Dey). DECaLS, BASS, and MzLS together include data obtained, respectively, at the Blanco telescope, Cerro Tololo Inter-American Observatory, National Optical Astronomy Observatory (NOAO); the Bok telescope, Steward Observatory, University of Arizona; and the Mayall telescope, Kitt Peak National Observatory, NOAO. The Legacy Surveys project is honored to be permitted to conduct astronomical research on Iolkam Du'ag (Kitt Peak), a mountain with particular significance to the Tohono O'odham Nation.

NOAO is operated by the Association of Universities for Research in Astronomy (AURA) under a cooperative agreement with the National Science Foundation.

The Legacy Survey team makes use of data products from the Near-Earth Object Wide-field Infrared Survey Explorer (NEOWISE), which is a project of the Jet Propulsion Laboratory/California Institute of Technology. NEOWISE is funded by the National Aeronautics and Space Administration.

The Legacy Surveys imaging of the DESI footprint is supported by the Director, Office of Science, Office of High Energy Physics of the U.S. Department of Energy under contract No. DE-AC02-05CH1123, by the National Energy Research Scientific Computing Center, a DOE Office of Science User Facility under the same contract; and by the U.S. National Science Foundation, Division of Astronomical Sciences under contract No. AST-0950945 to NOAO.

Funding for SDSS-III has been provided by the Alfred P. Sloan Foundation, the Participating Institutions, the National Science Foundation, and the U.S. Department of Energy Office of Science. The SDSS-III website is http://www.sdss3.org/.

SDSS-III is managed by the Astrophysical Research Consortium for the Participating Institutions of the SDSS-III Collaboration, including the University of Arizona, the Brazilian Participation Group, Brookhaven National Laboratory, Carnegie Mellon University, University of Florida, the French Participation Group, the German Participation Group, Harvard University, the Instituto de Astrofisica de Canarias, the Michigan State/Notre Dame/JINA Participation Group, Johns Hopkins University, Lawrence Berkeley National Laboratory, Max Planck Institute for Astrophysics, Max Planck Institute for Extraterrestrial Physics, New Mexico State University, New York University, Ohio State University, Pennsylvania State University, University of Portsmouth, Princeton University, the Spanish Participation Group, University of Tokyo, University 
of Utah, Vanderbilt University, University of Virginia, University of Washington, and Yale University.

Facilities: Spitzer (IRAC), HST (WFPC2, WFC3), Mayall (CCD Mosaic imager), Swift (UVOT), Hale (WIRC), PO:1.5 m (SEDM), Keck:I (LRIS), OANSPM:HJT (RATIR), UKIRT (WFCAM), Gemini:Gillett (GNIRS), EVLA, AMI.

\section{ORCID iDs}

Jacob E. Jencson (1) https://orcid.org/0000-0001-5754-4007 Mansi M. Kasliwal (i) https://orcid.org/0000-0002-5619-4938 Howard E. Bond (ib https://orcid.org/0000-0003-1377-7145 Joel Johansson (1) https://orcid.org/0000-0001-5975-290X Kunal P. Mooley (i) https://orcid.org/0000-0002-2557-5180 Frank J. Masci (i) https://orcid.org/0000-0002-8532-9395 Ann Marie Cody (i) https://orcid.org/0000-0002-3656-6706 Ori D. Fox (1) https://orcid.org/0000-0003-2238-1572 Robert D. Gehrz (1) https://orcid.org/0000-0003-1319-4089 Peter A. Milne (i) https://orcid.org/0000-0002-0370-157X Daniel A. Perley (i) https://orcid.org/0000-0001-8472-1996 Schuyler D. Van Dyk (i) https://orcid.org/0000-00019038-9950

\section{References}

Adams, S. M., Jencson, J. E., \& Kasliwal, M. M. 2016a, ATel, 9441 Adams, S. M., Kochanek, C. S., Prieto, J. L., et al. 2016b, MNRAS, 460, 1645 Alam, S., Albareti, F. D., Allende Prieto, C., et al. 2015, ApJS, 219, 12 Anderson, J. P., González-Gaitán, S., Hamuy, M., et al. 2014, ApJ, 786, 67 Baron, E., Branch, D., \& Hauschildt, P. H. 2007, ApJ, 662, 1148 Bessell, M. S., Castelli, F., \& Plez, B. 1998, A\&A, 333, 231 Blagorodnova, N., Kotak, R., Polshaw, J., et al. 2017, ApJ, 834, 107 Blagorodnova, N., Neill, J. D., Walters, R., et al. 2018, PASP, 130, 035003 Bode, M. F., \& Evans, A. 1980, MNRAS, 193, 21P

Bond, H. E., Bedin, L. R., Bonanos, A. Z., et al. 2009, ApJL, 695, L154 Bond, H. E., Henden, A., Levay, Z. G., et al. 2003, Natur, 422, 405 Borish, H. J., Huang, C., Chevalier, R. A., et al. 2015, ApJ, 801, 7 Botticella, M. T., Pastorello, A., Smartt, S. J., et al. 2009, MNRAS, 398, 1041 Botticella, M. T., Smartt, S. J., Kennicutt, R. C., et al. 2012, A\&A, 537, A132 Bowers, E. J. C., Meikle, W. P. S., Geballe, T. R., et al. 1997, MNRAS, 290, 663

Butler, N., Klein, C., Fox, O., et al. 2012, Proc. SPIE, 8446, 844610 Cappellaro, E., Botticella, M. T., Pignata, G., et al. 2015, A\&A, 584, A62 Cardelli, J. A., Clayton, G. C., \& Mathis, J. S. 1989, ApJ, 345, 245 Casali, M., Adamson, A., Alves de Oliveira, C., et al. 2007, A\&A, 467, 777 Cenko, S. B., Fox, D. B., Moon, D.-S., et al. 2006, PASP, 118, 1396 Chapman, N. L., Mundy, L. G., Lai, S.-P., \& Evans, N. J., II 2009, ApJ, 690, 496

Chevalier, R. A. 1982, ApJ, 259, 302

Chevalier, R. A. 1998, ApJ, 499, 810

Chevalier, R. A., \& Fransson, C. 2006, ApJ, 651, 381

Chevalier, R. A., Fransson, C., \& Nymark, T. K. 2006, ApJ, 641, 1029

Chomiuk, L., Soderberg, A. M., Chevalier, R. A., et al. 2016, ApJ, 821, 119 Ciatti, F., \& Barbon, R. 1971, MmSAI, 42, 145

Cohen, M., Wheaton, W. A., \& Megeath, S. T. 2003, AJ, 126, 1090

Cresci, G., Mannucci, F., Della Valle, M., \& Maiolino, R. 2007, A\&A, 462, 927

Davies, M. L., Franzen, T. M. O., Davies, R. D., et al. 2009, MNRAS, 400, 984 de Jager, C., Nieuwenhuijzen, H., \& van der Hucht, K. A. 1988, A\&AS, 72,259

Diamond, T. R., Hoeflich, P., \& Gerardy, C. L. 2015, ApJ, 806, 107

Draine, B. T., \& Lee, H. M. 1984, ApJ, 285, 89

Dwek, E. 1983, ApJ, 274, 175

Dwek, E. 1985, ApJ, 297, 719

Eisenstein, D. J., Weinberg, D. H., Agol, E., et al. 2011, AJ, 142, 72 Elitzur, M., \& Ivezić, Ž 2001, MNRAS, 327, 403

Ergon, M., Jerkstrand, A., Sollerman, J., et al. 2015, A\&A, 580, A142 Faran, T., Nakar, E., \& Poznanski, D. 2018, MNRAS, 473, 513 Fazio, G. G., Hora, J. L., Allen, L. E., et al. 2004, ApJS, 154, 10 Fitzpatrick, E. L. 1999, PASP, 111, 63

Fox, O. D., Chevalier, R. A., Dwek, E., et al. 2010, ApJ, 725, 1768
Fox, O. D., Chevalier, R. A., Skrutskie, M. F., et al. 2011, ApJ, 741, 7 Fox, O. D., \& Filippenko, A. V. 2013, ApJL, 772, L6

Fox, O. D., Johansson, J., Kasliwal, M., et al. 2016, ApJL, 816, L13 Gehrels, N., Chincarini, G., Giommi, P., et al. 2004, ApJ, 611, 1005

Gehrz, R. D. 1988, Natur, 333, 705

Gehrz, R. D., \& Ney, E. P. 1990, PNAS, 87, 4354

Gehrz, R. D., Roellig, T. L., Werner, M. W., et al. 2007, RScI, 78, 011302

Grossan, B., Spillar, E., Tripp, R., et al. 1999, AJ, 118, 705

Hildebrand, R. H. 1983, QJRAS, 24, 267

Hodgkin, S. T., Irwin, M. J., Hewett, P. C., \& Warren, S. J. 2009, MNRAS, 394, 675

Horesh, A., Kulkarni, S. R., Corsi, A., et al. 2013a, ApJ, 778, 63

Horesh, A., Stockdale, C., Fox, D. B., et al. 2013b, MNRAS, 436, 1258

Horiuchi, S., Beacom, J. F., Bothwell, M. S., \& Thompson, T. A. 2013, ApJ, 769, 113

Horiuchi, S., Beacom, J. F., Kochanek, C. S., et al. 2011, ApJ, 738, 154 Humphreys, R. M., Bond, H. E., Bedin, L. R., et al. 2011, ApJ, 743, 118 Ivezic, Z., \& Elitzur, M. 1997, MNRAS, 287, 799

Ivezic, Z., Nenkova, M., \& Elitzur, M. 1999, arXiv:astro-ph/9910475 Jencson, J. E., Adams, S., Kasliwal, M. M., et al. 2016, ATel, 9434 Jencson, J. E., Kasliwal, M. M., Johansson, J., et al. 2017, ApJ, 837, 167 Johansson, J., Goobar, A., Kasliwal, M. M., et al. 2017, MNRAS, 466, 3442 Jordi, K., Grebel, E. K., \& Ammon, K. 2006, A\&A, 460, 339 Kankare, E., Mattila, S., Ryder, S., et al. 2008, ApJL, 689, L97 Kankare, E., Mattila, S., Ryder, S., et al. 2012, ApJL, 744, L19 Kasliwal, M. M., Bally, J., Masci, F., et al. 2017, ApJ, 839, 88 Kennicutt, R. C., Jr. 1998, ARA\&A, 36, 189

Kochanek, C. S. 2011, ApJ, 741, 37

Kool, E. C., Ryder, S., Kankare, E., et al. 2018, MNRAS, 473, 5641

Kotak, R., Meikle, P., Pozzo, M., et al. 2006, ApJL, 651, L117

Kotak, R., Meikle, P., van Dyk, S. D., Höflich, P. A., \& Mattila, S. 2005, ApJL, 628, L123

Kotak, R., Meikle, W. P. S., Farrah, D., et al. 2009, ApJ, 704, 306

Laor, A., \& Draine, B. T. 1993, ApJ, 402, 441

Levesque, E. M., Massey, P., Olsen, K. A. G., et al. 2005, ApJ, 628, 973 Maguire, K., Di Carlo, E., Smartt, S. J., et al. 2010, MNRAS, 404, 981 Maiolino, R., Vanzi, L., Mannucci, F., et al. 2002, A\&A, 389, 84 Mannucci, F., Della Valle, M., \& Panagia, N. 2007, MNRAS, 377, 1229

Mannucci, F., Maiolino, R., Cresci, G., et al. 2003, A\&A, 401, 519

Martin, D. C., Chang, D., Matuszewski, M., et al. 2014, ApJ, 786, 106

Mathis, J. S., Rumpl, W., \& Nordsieck, K. H. 1977, ApJ, 217, 425

Mattila, S., Dahlen, T., Efstathiou, A., et al. 2012, ApJ, 756, 111

Mattila, S., \& Meikle, W. P. S. 2001, MNRAS, 324, 325

Mattila, S., Meikle, W. P. S., Lundqvist, P., et al. 2008, MNRAS, 389, 141

Mattila, S., Väisänen, P., Farrah, D., et al. 2007, ApJL, 659, L9

Matuszewski, M., Chang, D., Crabill, R. M., et al. 2010, Proc. SPIE, 7735 , $77350 \mathrm{P}$

Mauerhan, J. C., Smith, N., Filippenko, A. V., et al. 2013, MNRAS, 430, 1801 Meikle, W. P. S., Kotak, R., Farrah, D., et al. 2011, ApJ, 732, 109

Meikle, W. P. S., Spyromilio, J., Varani, G.-F., \& Allen, D. A. 1989, MNRAS, 238, 193

Miluzio, M., Cappellaro, E., Botticella, M. T., et al. 2013, A\&A, 554, A127 Nousek, J. A. 2004, Proc. SPIE, 5165, 169

Oke, J. B., Cohen, J. G., Carr, M., et al. 1995, PASP, 107, 375

Panagia, N., Van Dyk, S. D., Weiler, K. W., et al. 2006, ApJ, 646, 369

Pastorello, A., Cappellaro, E., Inserra, C., et al. 2013, ApJ, 767, 1

Pastorello, A., Sauer, D., Taubenberger, S., et al. 2006, MNRAS, 370, 1752

Pastorello, A., Smartt, S. J., Mattila, S., et al. 2007, Natur, 447, 829

Pastorello, A., Valenti, S., Zampieri, L., et al. 2009, MNRAS, 394, 2266

Pastorello, A., Zampieri, L., Turatto, M., et al. 2004, MNRAS, 347, 74

Patat, F., Cappellaro, E., Danziger, J., et al. 2001, ApJ, 555, 900

Perrott, Y. C., Scaife, A. M. M., Green, D. A., et al. 2013, MNRAS, 429, 3330

Prieto, J. L., Kistler, M. D., Thompson, T. A., et al. 2008, ApJL, 681, L9

Romero-Cañizales, C., Herrero-Illana, R., Pérez-Torres, M. A., et al. 2014, MNRAS, 440, 1067

Roming, P. W. A., Kennedy, T. E., Mason, K. O., et al. 2005, SSRv, 120, 95 Rubin, A., \& Gal-Yam, A. 2016, ApJ, 828, 111

Sanders, N. E., Soderberg, A. M., Gezari, S., et al. 2015, ApJ, 799, 208

Schlafly, E. F., \& Finkbeiner, D. P. 2011, ApJ, 737, 103

Schlegel, D. J., Finkbeiner, D. P., \& Davis, M. 1998, ApJ, 500, 525

Shappee, B. J., Stanek, K. Z., Kochanek, C. S., Holoien, T. W.-S., \& Prieto, J. L. 2016, ATel, 9446

Sheth, K., Regan, M., Hinz, J. L., et al. 2010, PASP, 122, 1397

Shostak, G. S. 1975, ApJ, 198, 527

Smith, N. 2014, ARA\&A, 52, 487

Smith, N., Andrews, J. E., Van Dyk, S. D., et al. 2016, MNRAS, 458, 950 
Smith, N., Li, W., Silverman, J. M., Ganeshalingam, M., \& Filippenko, A. V. 2011, MNRAS, 415, 773

Soderberg, A. M., Brunthaler, A., Nakar, E., Chevalier, R. A., \& Bietenholz, M. F. 2010, ApJ, 725, 922

Soderberg, A. M., Margutti, R., Zauderer, B. A., et al. 2012, ApJ, 752, 78

Sorce, J. G., Tully, R. B., Courtois, H. M., et al. 2014, MNRAS, 444, 527

Sparks, W. B., Bond, H. E., Cracraft, M., et al. 2008, AJ, 135, 605

Spyromilio, J., Gilmozzi, R., Sollerman, J., et al. 2004, A\&A, 426, 547

Szalai, T., \& Vinkó, J. 2013, A\&A, 549, A79

Szalai, T., Vinkó, J., Balog, Z., et al. 2011, A\&A, 527, A61

Szalai, T., Zsíros, S., Fox, O. D., Pejcha, O., \& Müller, T. 2018, ApJ, submitted (arXiv:1803.02571)

Szczygieł, D. M., Prieto, J. L., Kochanek, C. S., et al. 2012, ApJ, 750, 77

Takáts, K., \& Vinkó, J. 2006, MNRAS, 372, 1735

Tartaglia, L., Sand, D. J., Valenti, S., et al. 2018, ApJ, 853, 62
Thompson, T. A., Prieto, J. L., Stanek, K. Z., et al. 2009, ApJ, 705, 1364

Tinyanont, S., Kasliwal, M. M., Fox, O. D., et al. 2016, ApJ, 833, 231

Tylenda, R., Hajduk, M., Kamiński, T., et al. 2011, A\&A, 528, A114

Vacca, W. D., Cushing, M. C., \& Rayner, J. T. 2003, PASP, 115, 389

Valenti, S., Howell, D. A., Stritzinger, M. D., et al. 2016, MNRAS, 459, 3939

Van Dyk, S. D., \& Matheson, T. 2012, ASSL, 384, 249

Vinko, J., Varga-Verebelyi, E., \& Sarneczky, K. 2016, ATel, 9451

Watson, A. M., Richer, M. G., Bloom, J. S., et al. 2012, Proc. SPIE, 8444, 84445L

Werner, M. W., Roellig, T. L., Low, F. J., et al. 2004, ApJS, 154, 1

Wilson, J. C., Eikenberry, S. S., Henderson, C. P., et al. 2003, Proc. SPIE, 4841,451

Wooden, D. H., Rank, D. M., Bregman, J. D., et al. 1993, ApJS, 88, 477

Xiao, L., \& Eldridge, J. J. 2015, MNRAS, 452, 2597

Yuan, F., Jerkstrand, A., Valenti, S., et al. 2016, MNRAS, 461, 2003 